\title{
Design and characterization of visible-light LED sources for microstructured photoreactors
}

Anca Roibu ${ }^{a}$, Rishi Bharadwaj Morthala ${ }^{a}$, M. Enis Leblebicia ${ }^{a}$ Dorota Koziej ${ }^{b}$, Tom Van Gerven $^{a} \&$ Simon Kuhn ${ }^{\mathrm{a}, *}$

${ }^{a}$ KU Leuven, Department of Chemical Engineering, Celestijnenlaan 200F, 3001 Leuven, Belgium.

${ }^{b}$ ETH Zürich, Laboratory for Multifunctional Materials, Department of Materials, Vladimir-PrelogWeg 1-5/10, 8093 Zürich, Switzerland. Present address: University of Hamburg, Center for Hybrid Nanostructures (CHyN)

${ }^{*}$ Correspondence to S.K. (email: simon.kuhn@kuleuven.be)

\section{Abstract}

The design of stable, compact, and uniform LED light sources for continuous microstructured photoreactors is reported. The electrical and thermal properties of green LEDs are translated into an efficient control and cooling strategy. To study the irradiance uniformity and efficiency to irradiate the microfluidic channel, narrow viewing angle LEDs were configured in four arrays. The irradiance uniformity experienced by the microchannel is determined with an irradiance model, which is improved by using near-field goniophotometer measurements for small distances between the light source and reactor. Maximum light uniformity is achieved below the LED-reactor distance of $1.5 \mathrm{~cm}$. Exceeding this distance and employing arrays with a larger number of LEDs did not improve the uniformity on the microchannel. Furthermore, the energy efficiency of the photoreactor is quantified by combining near-field goniophotometer measurements, irradiance modeling and actinometry. It was shown that below $2 \mathrm{~cm}$ the photon losses were reduced when the LED positions matched the microchannel geometry, however a low utilization of the consumed electrical energy is observed irrespective of the LED array design. The characterization methodology presented in this study enables the identification and quantification of the limiting factors.

\section{Introduction}

Continuous microreactors are increasingly used for photochemical transformations due to their improved photon and mass transfer in comparison to batch reactors. ${ }^{1,2}$ They were previously combined with various light sources, such as conventional mercury lamps, compact fluorescence lamps (CFLs), halogen lamps and light-emitting diodes (LEDs). ${ }^{3}$ For operation in the UV range low-pressure and medium-pressure mercury lamps are commonly used. For processes in the visible range, LEDs are increasingly integrated with microreactors due to their monochromaticity, tunable power and small size. Moreover, no optical filters are required, and the electricity is converted directly into photons with desired wavelength. The visible-light LEDs were reported to have the lowest cost associated with the generation of photons in the visible range. ${ }^{4}$ Leblebici et al. compared 12 commonly used photo reactors for waste water treatment based on the photochemical space-time yield (PSTY), which relates the reactor space-time yield (STY) to the standardized lamp power. ${ }^{5}$ One of the compared reactor designs was a microstructured reactor 
with a volume of $1.5 \mu \mathrm{L}$. While this reactor had the largest STY, the PSTY did not compare favorably with other designs as it was illuminated by a 120 W UV lamp. Consequently, if the lamp power would be adjusted to the small illuminated volume, e.g. by using LEDs, the PSTY of the microreactor would be the highest among the evaluated reactor designs. The importance of geometrical compatibility between a photoreactor and its light source was also shown by Ziegenbalg et al. ${ }^{6}$ They investigated a system comprised of a glass microreactor equipped with Organic Light-Emitting Diodes (OLEDs) shaped as surface emitting panels. By using modeling and data obtained from photoreaction optimization, they found that $60 \%$ of the incoming photons were lost due to the area mismatch between the narrow reactor channels and the light source. The possibility to choose between LEDs of various sizes, shapes and radiation patterns allows flexibility in designing light sources which match the geometry of microreactors. In most cases, multiple LEDs are required to achieve the photon flux necessary for operating the photoreactor at an optimum productivity. The number and the geometrical and electrical arrangement of the LEDs in the light source array will determine the photon flux received by the reactor and the irradiance uniformity on the reactor surface. The distribution of the light on the reactor surface has to be considered, as decreased photonic efficiency of photocatalytic reactions was attributed to the inhomogeneity of the LED arrays. ${ }^{7,8}$ Casado et al. investigated the irradiance uniformity on a catalyst surface with a diameter of $6 \mathrm{~cm}$ in a batch photoreactor for LED arrays equipped with LEDs with a broad viewing angle of $85^{\circ} .{ }^{9}$ The variation of the homogeneity (relative standard deviation of the irradiance) was determined using discrete ordinate method (DOM) and the distance between the LEDs and the catalytic surface was identified as the main parameter affecting the uniformity. It was found that the uniformity increases with the distance at the expense of the photon flux reaching the catalytic surface. At a distance of $4 \mathrm{~cm}$ the variation of uniformity was around $26 \%$, and $80 \%$ of the incident light reached the catalyst, while at $8 \mathrm{~cm}$ the irradiance uniformity varied by $7.5 \%$, but only $30 \%$ of the incident light reached the catalyst, the rest being lost in the planar geometry.

The strategy for designing LED arrays for the irradiation of microreactors is different than for batch reactors. Firstly, the distance between the light source and the reactor where the irradiance should be uniform needs to be minimal. As the required productivity is usually achieved by numbering up microreactors, the photoreactor system should have a small footprint. Secondly, a higher photon loss is expected when irradiating microfluidic channels compared to irradiating a wide reactor surface. Therefore, LEDs with focused emission would be more suitable than wide angle LEDs. However, implementing this strategy for designing novel LED light sources is limited by the accuracy of the currently available modeling tools and experimental measurements at short distance between the LEDs and the irradiated reactor (below $5 \mathrm{~cm}$ ), and especially for narrow viewing angle LEDs as reported by Kheyrandish et al. ${ }^{10,11}$.

The light emission is also strongly affected by the driving mode of the LEDs which is seldomly studied in light source design for photoreactors. The radiant flux and the spectral distribution depend directly on the forward current, therefore a constant current has to be ensured through the LEDs. This was realized for a single LED by using a constant current driver controlled by a potentiometer ${ }^{10}$, however the current control becomes more complex when an entire LED array is powered. Sergejevs et al. designed a light source consisting of 36 UV-LEDs for the illumination of a catalyst surface in a batch reactor. ${ }^{12}$ Series containing 3 LEDs were current driven and calibrated to account for the variability in the electrical and optical properties of the LEDs due to 
the manufacturing process. As $70 \%$ of the electrical power consumed by the LEDs was converted into heat, a temperature sensor and a liquid cooling system were used to avoid overheating of the printed circuit board (PCB). Such overheating can lead to a decrease in the optical output and a red-shift of the dominantly emitted wavelength. Kheyrandish et al. ${ }^{10}$ reported a decrease of $29 \%$ in the emission of a single UV-LED when the solder temperature increased by $36^{\circ} \mathrm{C}$ and Casado et al. ${ }^{9}$ observed a decrease of $20 \%$ for a variation of $40^{\circ} \mathrm{C}$ in the temperature of a 36 UV-LED circuit board.

Above discussion highlights that multiple parameters have to be simultaneously considered when designing LED arrays to be used as light sources in photochemical processes. Here, the rational design and quantitative characterization of visible-light LED light sources for continuous microstructured reactors is described. LEDs with narrow viewing angle are used to achieve an efficient irradiation of the microchannel. The electrical, optical and thermal properties of the LEDs are investigated and are related to the final designs of the LED boards. The arrangement and density of the LEDs on the LED board were varied, and the obtained designs were characterized in terms of uniformity using an irradiance model. The irradiance model at small distances between the LED board and the reactor is improved using near-field goniophotometer measurements, a procedure reported here for the first time. The maximum uniformity was reached at a LED-reactor distance below $1.5 \mathrm{~cm}$. The irradiance simulations show that increasing the density of the LEDs on the PCB or increasing the distance between the light source and reactor exceeding an optimal value do not improve the uniformity. Furthermore, the efficiency to transport the emitted photons into the microfluidic channel is quantified using the near-field goniophotometer measurements, irradiance model and actinometry. Our study confirms that for a flat plate microreactor the energy utilization is low for all LED arrays and continues to constitute a design challenge. Nevertheless, the presented toolbox and procedure will enable to increase the compatibility of the microreactor with the LED light sources for future continuous microstructured photoreactors.

\section{Experimental}

\subsection{Measurement of electrical and optical characteristics of single LEDs}

The LEDs (HLMP-CM3A-Z10DD) used in this study are through-hole LEDs, with clear epoxy encapsulation, and a diameter of $5 \mathrm{~mm}$ (obtained from Avago, which is currently Broadcom). According to the manufacturer datasheet ${ }^{13}$, the viewing angle is $30^{\circ}$, the typical forward voltage, $V_{F}$, is $3.2 \mathrm{~V}$ at a forward current, $I_{F}$, of $20 \mathrm{~mA}$ and the maximum $I_{F}$ is $30 \mathrm{~mA} .{ }^{13}$ The maximum emission wavelength is $516 \mathrm{~nm}$ at $20 \mathrm{~mA}$.

The characteristic $I_{F}$ vs. $V_{F}$ was measured for single LEDs using one digital multimeter placed in parallel (UNI-T) and another digital multimeter connected in series with the LED. The LED was powered by a DC lab power supply (Velleman). The spectral distributions at different values of $I_{F}$ ranging from 0-35 $\mathrm{mA}$ were measured by placing a cosine corrector (Avantes) at $1 \mathrm{~cm}$ distance from the tip of the LED. The cosine corrector was connected to a compact spectrometer (Ultra Low Straylight Fiber Optic UV/VIS/NIR spectrometer 200-1100 nm, Avantes) using an optical fiber (400 $\mu \mathrm{m}$ UV/VIS fiber, Avantes). 


\subsection{Manufacturing of LED light sources}

The microreactor (Chemtrix Inc.) used in this study consists of a serpentine channel in a borosilicate glass plate with an internal diameter of $0.1 \mathrm{~cm}$, length of $69.95 \mathrm{~cm}$ and total volume of $0.55 \mathrm{~mL}$. 2 LED light sources were designed and built in collaboration with the electrical workshop from the Department of Electrical Engineering, KU Leuven. The first light source contains LEDs placed at a LED-LED distance, $s$, of $0.8 \mathrm{~cm}$ and contains 144 LEDs. A channel configuration (CC-8mm) is achieved when $84 \mathrm{LEDs}$ are powered to match the serpentine reactor channel layout, and a matrix configuration (MC-8mm) is achieved when all of the 144 LEDs are powered. The second LED light source is characterized by $s=0.65 \mathrm{~cm}$, with the channel configuration (CC-6.5mm) of 102 LEDs and the matrix configuration (MC-8mm) of 177 LEDs. The driving board was realized in collaboration with the electrical workshop from the Department of Electrical Engineering, KU Leuven, and it controls the current through LED series using a current mirror. The design and operation of the driving board are presented in section 7 of the Electronic Supplementary Information (ESI).

\subsection{Thermal imaging analysis}

The thermal imaging analysis was realized in collaboration with the Laboratory for Multifunctional Materials, Department of Materials, ETH Zürich, Switzerland. Thermal images of single LEDs and LED boards were acquired using an infrared camera (Flir SC7650E) and analyzed using the FLIR RESEARCHIR MAX 4 software. The LEDs were covered with black paint so that the emissivity of the investigated materials can be assumed to equal 1. For acquiring the temperature profiles at different distance from the LED junction, the LED encapsulation was cut at various crosssections utilizing FORCIPOL polishers. The LEDs were powered by a DC lab power supply and the current through the LEDs was monitored using a digital multimeter.

\subsection{Near-field goniophotometer measurements and ray tracing}

Near-field goniophotometer measurements (NFGM) were used to quantify the radiant flux of a light source and the spatial distribution of the emitted light. NFGM for a single LED was acquired by the Light \& Lighting Laboratory (KU Leuven, Gent, Belgium). The LED was operated at a current of $8 \mathrm{~mA}$. NFGM were performed for a polar angle between 0 and $130^{\circ}$ with a resolution of $1^{\circ}$ and for an azimuthal angle between 0 and $360^{\circ}$ with a resolution of $1^{\circ}$. NFGM for the final designed light sources CC-8mm and MC-8mm configurations were performed by Laser 2000 (Photometric \& Optical Testing Services LLP, UK) and were calibrated with a spectroradiometer. The near-field measurement involves a photometer (camera) and a goniometer frame where the light source is placed. The goniometer frame moves at different angles and, simultaneously, the photometer acquires images of the light source. Then, the images are converted to a model, based on which a ray file is created. In the current work, the ray sets containing 10 million rays were imported in the ray tracing software FRED (Photon Engineering). The irradiance distribution on planes placed parallel to the light board were obtained by creating analysis surfaces at desired distances from the light source. For the single LED, the analysis surface was $4 \mathrm{~cm} \times 4 \mathrm{~cm}$ with a resolution between data points of $0.04 \mathrm{~cm}$. For the $\mathrm{CC}-8 \mathrm{~mm}$ and $\mathrm{MC}-8 \mathrm{~mm}$, the analysis surfaces were $12.5 \mathrm{~cm} \times 14.2 \mathrm{~cm}$ with a resolution of $0.02 \mathrm{~cm}$. For determining the variation of the irradiance in function of the polar angles, a spherical detector was used. The irradiance was considered between the polar angles ranging from $-90^{\circ}$ to $90^{\circ}$ and the azimuthal angles between $-180^{\circ}$ and $180^{\circ}$. 


\subsection{Actinometry}

The actinometric measurements were performed using the photo cycloreversion reaction of the diarylethene derivative, 1,2-bis(2,4-dimethyl-5-phenyl-3-thienyl)perfluorocyclopentene (DAE), from its closed form (DAE CF) to the open form (DAE OF). The solution of DAE CF is obtained by irradiating DAE OF (TCI Chemicals) solution in hexane (Biosolve) at $311 \mathrm{~nm}$. The detailed description of the measurement methodology was previously reported ${ }^{14}$ and the calculation of the photon flux is described in section 1 of the ESI. The scheme of the experimental set-up used for performing actinometric measurements is illustrated in Fig. 1.

The experimental set-up is placed in a fume hood which is covered with black fabric to avoid the exposure of the actinometer solutions to the ambient light. A mixture of DAE CF and DAE OF is fed through the microreactor using a syringe pump (Model Fusion 720, Chemyx Inc.) and PFA tubing (internal diameter of $0.5 \mathrm{~mm}$ ). The geometry of the microstructured reactor characterized by a serpentine channel with internal diameter of $1 \mathrm{~mm}$ can be observed in Fig.1. The syringe and tubes are covered with black material during actinometric measurements, only the glass reactor being exposed to the emission of the green LEDs. The liquid exiting the reactor flows through a cross-cell (Avantes) for on-line analysis. A balanced Deuterium-Halogen light source (215-2500 nm, Avantes) and a compact spectrometer (Ultra Low Straylight Fiber Optic UV/VIS/NIR spectrometer 200-1100 nm, Avantes) are connected to the flow cell through optical fibers (400 $\mu \mathrm{m}$ UV/VIS fiber, Avantes). The absorbance measurements are characterized by an integration time of $1.31 \mathrm{~ms}$ and 500 scans/measurement. The absorbance at $565-566 \mathrm{~nm}$ is continuously monitored using Avasoft 8 software.

Two thermocouples (Omega) are inserted in T-junctions which are placed near the inlet and outlet of the reactor to monitor the temperature of the liquid. The experiments were performed at room temperature, which varied between $24-28^{\circ} \mathrm{C}$. However, no temperature difference was observed between the outlet and inlet, and therefore the photoreaction was performed at isothermal conditions. The exiting actinometer solution was collected in a brown bottle and reused directly or after its exposure to UV light. 


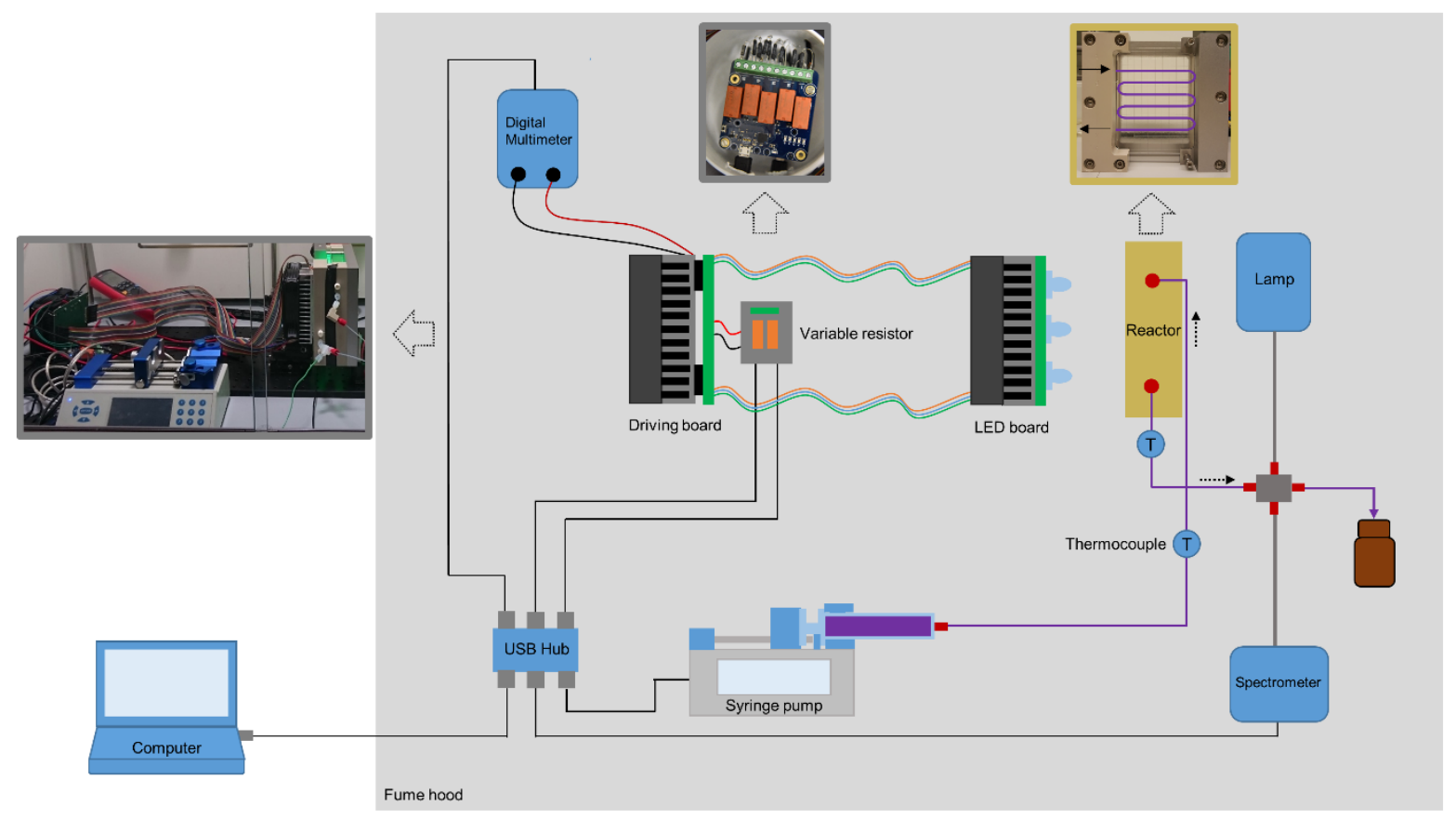

Figure 1. Schematic representation of the experimental setup for actinometric measurements. The experimental setup is placed in a fume hood which is illustrated by the gray area. The purple line indicates the flow of the actinometer solution from the syringe to the collection bottle. The syringe pump, the spectrometer, the variable resistor of the driving board and the digital multimeter are controlled by a computer outside the fume hood. The inset images show the side view of the set-up (syringe pump, driving and LED boards and reactor), the top view of the variable resistor and the front view of the reactor, respectively.

\section{Irradiance model}

\subsection{Theoretical considerations}

Two regions are distinguished in the radiometric modeling of a light source, i.e. the near-field and the far-field. In the near-field the light source is located close to the irradiated surface and is modeled as an extended area. In the far-field region, the light source is positioned at a far distance relative to the irradiated surface and is modeled as a point source. ${ }^{15-17}$ Usually, the distance where the far-field starts is approximated as 5 times the largest dimension of the light source. As an illustrative example, it was reported that for a LED with a $1 \mathrm{~mm}^{2}$ chip the far-field should start at $5 \mathrm{~mm}$, but in practice it occurs only after $20 \mathrm{~mm} .{ }^{16}$ Moreover, it was shown that the differentiation between the regions depends on the light distribution. If the LED is characterized by a narrow light distribution, the far-field is established at larger distances compared to LEDs with broad radiation patterns. ${ }^{17}$

The difference between these regions is illustrated in Fig.2a which represents the emission of multiple LEDs at different distances. As it can be observed, the resulting radiation pattern in the 
near-field varies with the distance between the source and irradiated surface, whereas in the far-field the radiation pattern does not depend on the distance.

The radiation field of a light source is usually modeled for the far-field region. Considering that the LED is a point source and is characterized by a Lambertian distribution pattern, the radiant intensity varies as a cosine function of the polar angle, $\theta[\mathrm{rad}] .{ }^{18}$

$$
I=I_{\mathrm{Max}} \cos ^{m} \theta
$$

where $I_{\mathrm{Max}}\left[\mathrm{W} \mathrm{sr}{ }^{-1}\right]$ is the maximum radiant intensity, located at the polar angle of $\theta=0$ and $m$ is a parameter depending on the LED encapsulation and it accounts for the fact that LEDs are not perfect Lambertian light sources. $m$ can be determined as follows: ${ }^{19}$

$$
m=\frac{-\ln 2}{\ln \left(\cos \theta_{1 / 2}\right)}
$$

where $\theta_{1 / 2}$ is the angle where the intensity decreases to half the value at $\theta=0$. The viewing angle of a LED is equal to $2 \theta_{1 / 2}$. For $m=1$, the light source is nearly a perfect Lambertian emitter.

The irradiance on a plane, $E\left[\mathrm{~W} \mathrm{~m}^{-2}\right]$, irradiated by one LED is related to the radiant intensity via

$$
E=\frac{I}{d^{2}}
$$

where $d$ is the distance from the light source to the irradiated surface.

a)

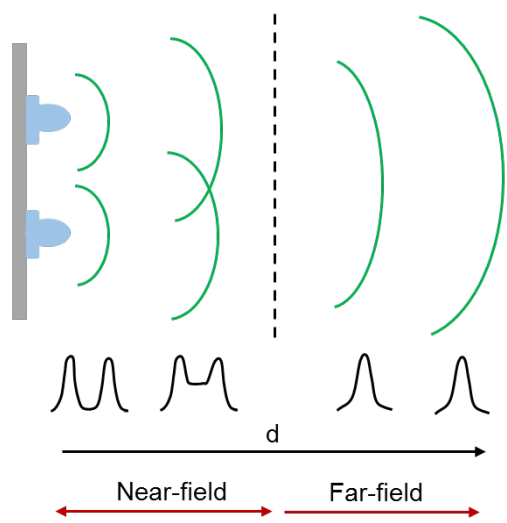

b)

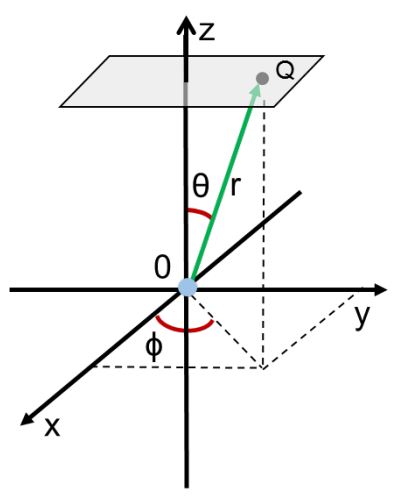

Figure 2. a) Illustration of the near-field and far-field for a LED array. The resulting radiation pattern in the near-field varies with the distance, $d$, between the source and irradiated surface, whereas in the far-field the radiation pattern does not depend on $d$. Adapted from ${ }^{17}$. b) Coordinate system used for the calculation of irradiance in an arbitrary point Q. $x, y, z$ are the Cartesian coordinates. $r$ is the radial coordinate, $\theta$ is the polar angle and $\phi$ is the azimuthal angle. 
The irradiance in any point on a target surface can be calculated considering Eq. (3). For example, the irradiance in the point $Q$ as represented in Fig. $2 b$ is:

$$
E(r, \theta, \phi)=\frac{I(\theta, \phi) \cos \theta}{r^{2}}
$$

For planar reactors, as also considered in this work, it is more convenient to express Eq. (4) in Cartesian coordinates, for which the irradiance in the point $Q$ becomes: ${ }^{16}$

$$
E(x, y, z)=\frac{z^{m}}{\left(x^{2}+y^{2}+z^{2}\right)^{\frac{m+2}{2}}} I(\theta, \phi)_{\mathrm{A}}
$$

where $x, y, z$ are the Cartesian coordinates as defined in Fig. 2 b. $\theta$ [rad] is the polar angle and $\phi$ [rad] is the azimuthal angle and can be calculated as:

$$
\begin{aligned}
& \theta(x, y, z)=\arctan \left(\frac{\sqrt{x^{2}+y^{2}}}{z}\right) \\
& \phi(x, y)=\arctan \left(\frac{y}{x}\right)
\end{aligned}
$$

$I(\theta, \phi)_{\mathrm{A}}\left[\mathrm{W} \mathrm{sr}{ }^{-1}\right]$ is the absolute radiant intensity which can be calculated using the radiant flux of the LED, $\Phi_{0}[\mathrm{~W}]$, and the normalized intensity distribution $I(\theta, \phi)^{16}$ :

$$
I(\theta, \phi)_{\mathrm{A}}=\frac{\Phi_{0}}{\int_{4 \pi} I(\theta, \phi) \mathrm{d} \Omega} I(\theta, \phi)
$$

Where $\mathrm{d} \Omega$ is the differential solid angle [sr] which is equal to

$$
\mathrm{d} \Omega=\sin \theta \mathrm{d} \theta \mathrm{d} \phi
$$

For the LEDs used in the current study, the intensity is not a function of the azimuthal angle, $\phi$. Consequently, at any given polar angle the intensity is the same for all the azimuthal angles, and Eq. (8) simplifies to:

$$
I(\theta)_{\mathrm{A}}=\frac{\Phi_{0}}{\int_{\theta_{1}}^{\theta_{2} \phi_{2}} \int_{\phi_{1}} I(\theta) \sin \theta \mathrm{d} \theta \mathrm{d} \phi} I(\theta)
$$


After integration over the azimuthal angles from 0 to $2 \pi$ and integration over the polar angles from 0 to $\frac{\pi}{2}$ the absolute intensity is calculated as:

$$
I(\theta)_{\mathrm{A}}=\frac{\Phi_{0}}{2 \pi \int_{0}^{\frac{\pi}{2}} I(\theta) \sin \theta \mathrm{d} \theta} I(\theta)
$$

\subsection{Irradiance model validation}

The design of compact light sources requires to simulate the irradiance at small distances. However, in the near-field region the LED cannot be considered as a point source, and therefore the radiant intensity and radiant intensity distribution cannot be used. It was previously shown that the values obtained at small distances differ from the ones determined in the far-field. ${ }^{10,20,21}$ To model the irradiance in both near- and far-field, this study proposes to replace the normalized radiant intensity, $I(\theta)$ in Eq. (11), with the normalized irradiance distribution $E(\theta)$. In the near-field, a different $E(\theta)$ will be used for each distance between the tip of the LED and the illuminated surface, $D$, whereas in the far-field, $E(\theta)$ does not varies with this distance $D$. The irradiance on a parallel surface irradiated by a LED located in the origin of the coordinate system becomes:

$$
E(x, y, z)=\frac{z}{\left(x^{2}+y^{2}+z^{2}\right)^{\frac{3}{2}}} \frac{\Phi_{0} E(\theta)}{2 \pi \int_{0}^{\frac{\pi}{2}} E(\theta) \sin \theta \mathrm{d} \theta}
$$

The angular irradiance distributions $E(\theta)$ were obtained using the ray sets generated from NFGM and the ray tracing software FRED. As illustrated in Fig. 3a, the rays originating from the LED were captured on a spherical detector surface created in FRED. An important aspect to consider is that the origin of the light source is identical to the center of the spherical detector. Manninen et al. reported that for some LEDs, the point source is located on the LED chip, and not at the tip of the LED ${ }^{22}$. In our case, $z$ is defined as:

$$
z=\Delta d+D
$$

where $\Delta d$ is the distance between the LED chip and the LED tip, which is equal to $0.46 \mathrm{~cm}$ for the type of LEDs used in this study.

Fig. $3 b$ illustrates an example of the irradiance profile as a function of the polar angle, $\theta$, and azimuthal angle, $\phi$. A slight asymmetry in the irradiance distribution subject to the angle $\phi$ is observed which can be explained by a misalignment of the LED chip during the manufacturing process. Theoretically a symmetric radiation pattern is expected, and therefore the irradiance variation was extracted as a function of $\theta$ for $\phi=0$. 


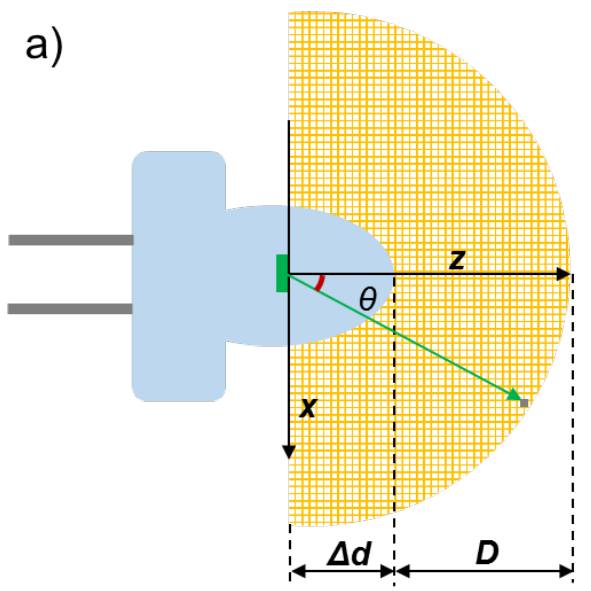

b)

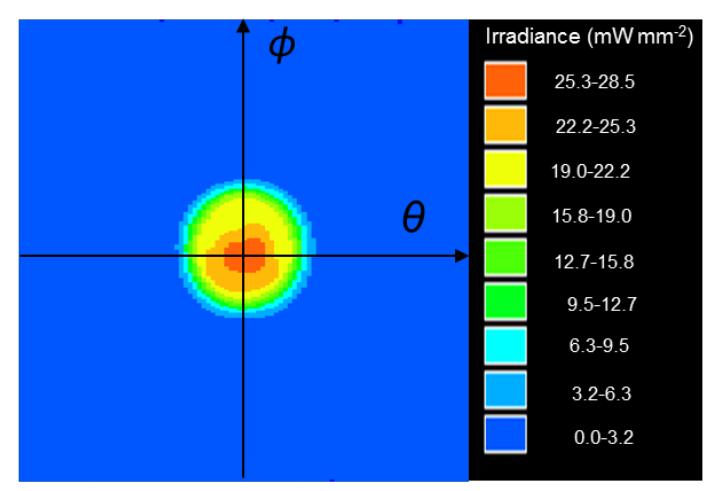

Figure 3. a) Geometry of the LED and of the spherical detector surface used in the ray tracing software to determine the angular irradiance distribution. $\Delta d$ denotes the distance between the LED chip and the tip of the LED, $D$ is the distance between the tip of the LED and the center of the detector surface and $\theta$ is the polar angle. b) Angular distribution of irradiance as a function of the polar angle, $\theta$, and azimuthal angle, $\phi$, extracted using the spherical detector surface for $D=1 \mathrm{~cm}$.

The extracted irradiance profiles were normalized by the maximum irradiance and fitted by nonlinear regression to a series of 8 Gaussian distributions according to:

$$
E(\theta)=\sum_{i=1}^{8} a_{i} e^{-\left(\frac{\theta-b_{i}}{c_{i}}\right)^{2}}
$$

where $a_{i}, b_{i}$ and $c_{i}$ are the fitting coefficients and $\theta$ is the polar angle [rad]. Therefore, for each distance, $D, E(\theta)$ is fitted using 24 coefficients. The nonlinear regression is characterized by coefficients of determination, $R^{2}$, larger than 0.999 , and the fitted values of the coefficients $a_{i}, b_{i}$, and $c_{i}$, are listed in Tab. S4. Using this procedure, $E(\theta)$ was determined in the range of $D=0.1 \mathrm{~cm}$ to $D=4 \mathrm{~cm}$. Figure 4 depicts the distributions obtained for distances between $0.1 \mathrm{~cm}$ and $2 \mathrm{~cm}$ in comparison to the distribution reported in the manufacturer datasheet (DS) representing the far-field region. It is clearly observed that the width of the irradiance distribution depends on the distance between the LED tip and the irradiated surface, which is attributed to the fact that at small distances, the LED chip is observed as an extended light source. The viewing angle at $E(\theta)=0.5$ increases from $31^{\circ}$ in the far-field (DS) until $60^{\circ}$ at $D=0.1 \mathrm{~cm}$. In addition, secondary 
peaks are observed at small distances $(D=0.1 \mathrm{~cm}$ and $D=0.5 \mathrm{~cm})$, which is in accordance with an observation by Manninen et al. ${ }^{22}$.

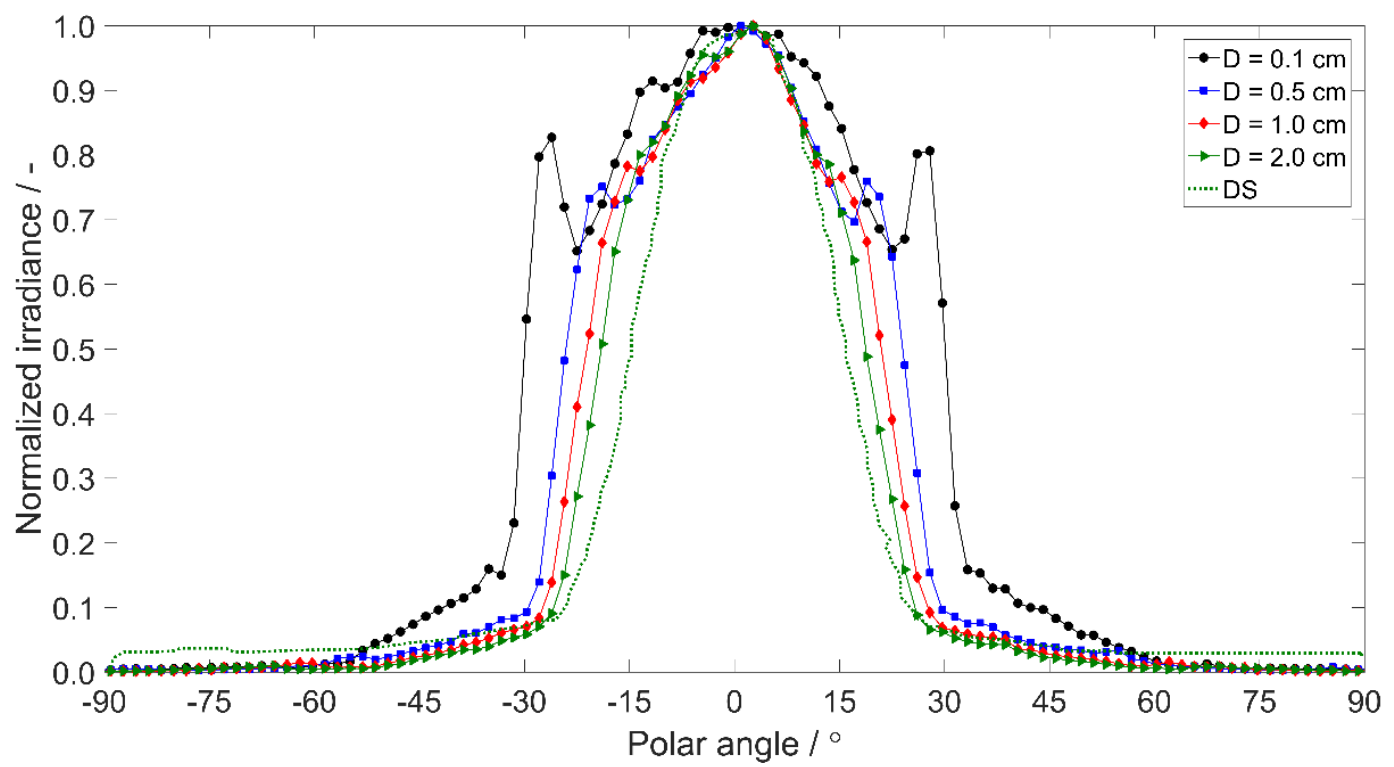

Figure 4. Variation of normalized irradiance, $E(\theta)$, in function of the polar angle, $\theta$, at different distances, $D$, between the tip of the LED and the center of the analysis surface. The angular normalized distributions are extracted from NFGM using a ray tracing software. DS denotes the normalized irradiance distribution extracted from the manufacturer datasheet, representing the far-field region.

The broadening of the angular irradiance distribution is quantified by the ratio, $R$, defined as:

$R=\frac{\Delta \theta_{1 / 2}(\text { Ray tracing })}{\Delta \theta_{1 / 2}(\mathrm{DS})}$

where $\Delta \theta_{1 / 2}$ (Ray tracing) is the width of the radiation pattern extracted from NFGM using ray tracing at $E(\theta)=0.5$, and $\Delta \theta_{1 / 2}$ (DS) is the width of the radiation pattern extracted from the datasheet at $E(\theta)=0.5$.

As it can be observed from Tab. 1, $R$ decreases sharply between $D=0.1 \mathrm{~cm}$ and $D=2 \mathrm{~cm}$, followed by a slight variation in the distance range from $2-4 \mathrm{~cm}$, which indicates the transition from the near-field to the far-field region. 
Table 1. Variation of $R$ values in function of the distance, $D$, between the tip of the LED and the center of the analysis surface.

\begin{tabular}{ll}
\hline$D / \mathrm{cm}$ & $R$ \\
\hline 0.1 & 1.94 \\
0.5 & 1.56 \\
1.0 & 1.37 \\
1.5 & 1.28 \\
2.0 & 1.24 \\
2.5 & 1.20 \\
3.0 & 1.18 \\
3.5 & 1.16 \\
4.0 & 1.15 \\
\hline
\end{tabular}

The emission broadening observed in the near-field will affect the irradiance profile on the reactor. Therefore, the irradiance was simulated on a parallel plane located at different $z$ and $D$ values by Eq. (12). Firstly, $E(\theta)$ extracted from NFGM is used and the model is denoted as model (NFGM). Secondly, $E(\theta)$ extracted from manufacturer datasheet is used and the model is denoted as model (DS). In both cases, a nearly Lambertian light source is assumed, therefore the parameter $m$ is equal to 1 . As the aim is the comparison of the normalized irradiance profiles, an arbitrary value was given for the radiant flux, $\Phi_{0}$. In order to assess the accuracy of the two models, the irradiance values extracted along the $x$ coordinate at $y=0 \mathrm{~cm}$ are compared with the irradiance resulted from the ray tracing software. The geometries of the LED and analysis surface employed in the ray tracing software are shown in Fig. 5.

a)

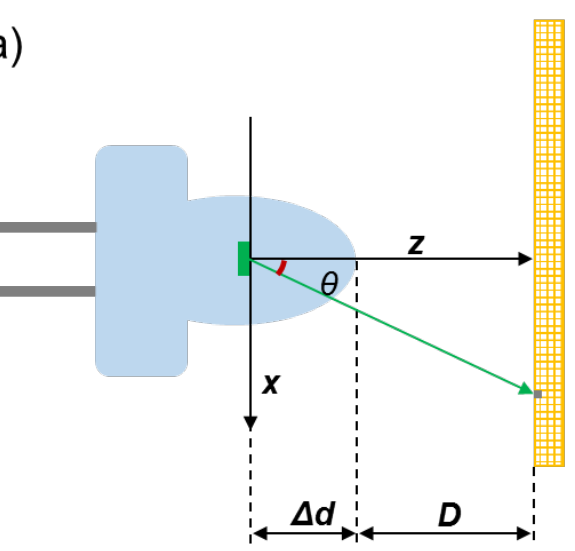

b)

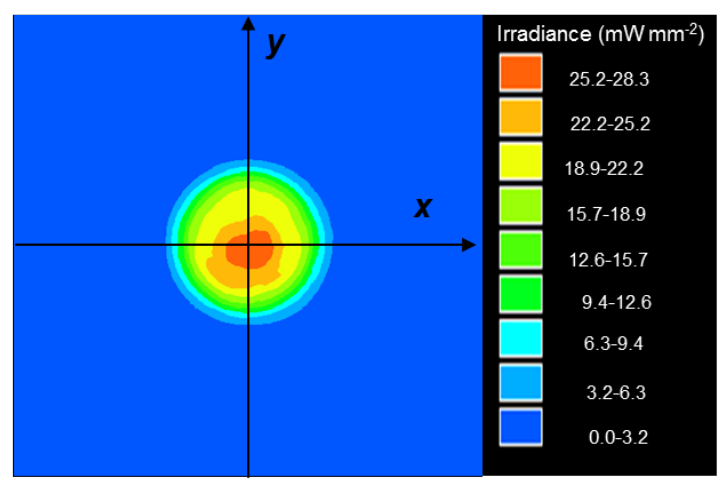

Figure 5. a) The geometry of the LED and of the analysis surface used in the ray tracing software to determine the variation of irradiance on a parallel plane at various $z$. $\Delta d$ is the distance between the LED chip and the tip of the LED, $D$ is the distance between the tip of the LED and the center of the analysis surface and $\theta$ is the polar angle. b) Irradiance distribution in function of the coordinates $x$ and $y$ extracted on an analysis surface for $D=1 \mathrm{~cm}$. 
The resulting irradiance profiles for distances between $D=0.1 \mathrm{~cm}$ and $D=2 \mathrm{~cm}$ are compared in Fig. 6. The irradiance values obtained using the model proposed in this work (model (NFGM)) are in a good agreement with the irradiance obtained by ray tracing. As expected, the model based on the manufacturer datasheet (model (DS)) underestimates the width of the LED emission at short distances and therefore the application of model (DS) is not recommended especially at small distances. For the remainder of this paper model (NFGM) will be used to quantify the irradiance uniformity and the irradiance on the microchannel surface.

a)

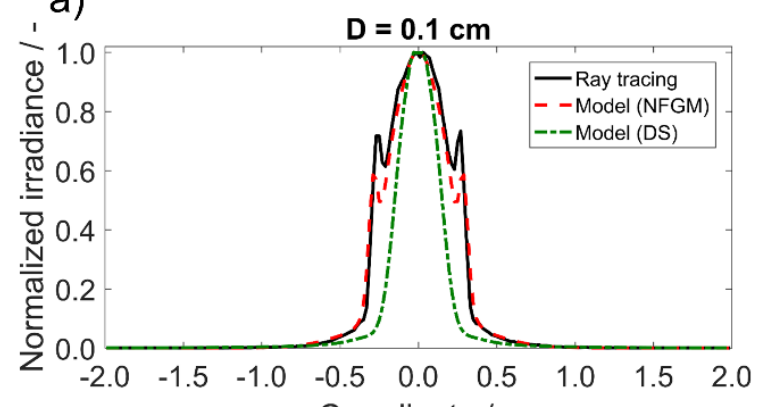

c)

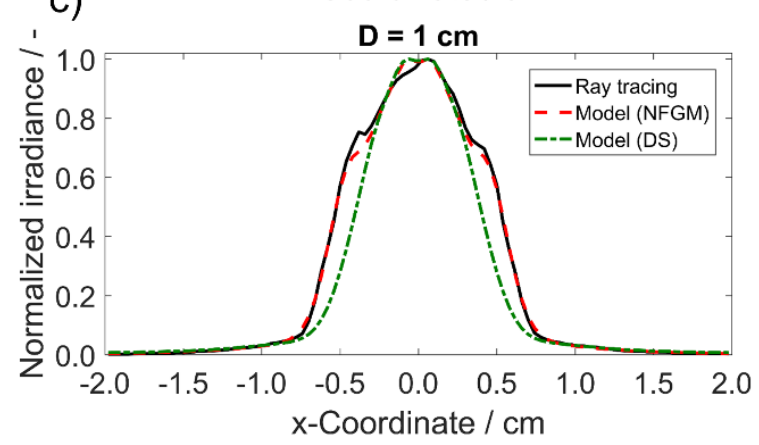

b)
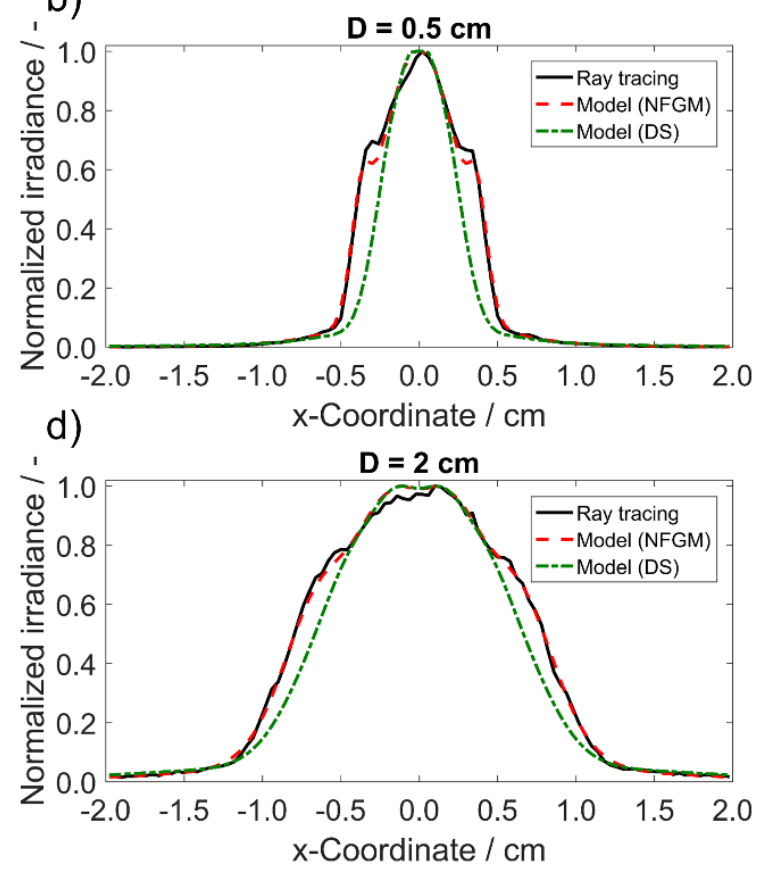

Figure 6. Comparison between the irradiance extracted from ray tracing software and predicted using model (NFGM) and model (DS). The comparison is realized at $y=0 \mathrm{~cm}$ and at a) $D=0.1 \mathrm{~cm}$, b) $D=0.5 \mathrm{~cm}$, c) $D=1 \mathrm{~cm}$, d) $D=2 \mathrm{~cm}$. $D$ is the distance between the tip of the LED and the center of the analysis surface.

\subsection{Absolute irradiance modeling on the reactor surface irradiated by an LED array}

To calculate the irradiance on the reactor surface, the irradiance on a plane parallel to the designed LED arrays needs to be simulated, by considering the contribution of all LEDs according to:

$$
E(x, y, z)=\sum_{M} \frac{z}{\left(\left(x-x_{0}\right)^{2}+\left(y-y_{0}\right)^{2}+z^{2}\right)^{\frac{3}{2}}} \frac{\Phi_{0} E(\theta)}{2 \pi \int_{0}^{\frac{\pi}{2}} E(\theta) \sin \theta \mathrm{d} \theta}
$$

where $M$ is the number of LEDs of the investigated array and $x_{0}, y_{0}$ are the coordinates of the LEDs. $\Phi_{0}$ denotes the radiant flux of one LED, which was calculated by dividing the measured 
radiant flux (using a near-field goniophotometer calibrated with a spectroradiometer) by the number of LEDs in the array.

\section{Results and discussion}

\subsection{Design of the LED boards}

\subsubsection{LED array layout}

The main criteria considered during the LED array design were efficiency, spatial homogeneity of irradiance on the reactor surface and optical output stability. As previously discussed, more than $30 \%$ of the consumed electrical power by an LED is converted to photons, the rest being lost as heat. When the incident photons are used efficiently, a lower number of LEDs are required and consequently a lower amount of heat needs to be dissipated. In the case of microreactors, the surface and volume exposed to the light are low, therefore only a small portion of the incident photons are absorbed by the reaction mixture. In order to minimize the radiation losses, the geometry of the light source should be compatible with the microreactor geometry. The microreactor consists of a serpentine channel with circular cross-section (internal diameter of $1 \mathrm{~mm}$ ) etched in a borosilicate glass plate. The geometry of the reactor channel is illustrated in Fig. 7 with a blue line. To compare the efficiency to deliver photons to the microchannel depending on the layout of the LED array, two configurations were considered: channel configuration (CC) and matrix configuration (MC). The CC board mimics the geometry of the microreactor channel, and $\mathrm{MC}$ is based on $\mathrm{CC}$ with additional LEDs placed between the reactor channels.

The distance between LEDs, $s$, which leads to a homogeneous irradiance distribution for a linear array (> 4 LEDs) can be calculated as reported by Moreno et al. ${ }^{19}$ :

$$
s=\sqrt{\frac{3.2773}{m+4.2539}} z
$$

where $m$ was calculated according to Eq. 2. Using LEDs characterized by a narrow viewing angle, $2 \theta_{1 / 2}$, of $30^{\circ}$ a homogeneous illumination at a distance $z=2 \mathrm{~cm}$ can be obtained if the LEDs are placed at a distance $s$ of $7.3 \mathrm{~mm}$. In order to investigate the influence of the LED density in addition to the LED array layout, two LED boards were designed with a distance between LEDs $s$ of $6.5 \mathrm{~mm}$ (CC \& MC configuration) and $8 \mathrm{~mm}$ (CC \& MC configuration) respectively. The layouts of the designed LED arrays are compared with the reactor geometry in Fig. 7. 
a)

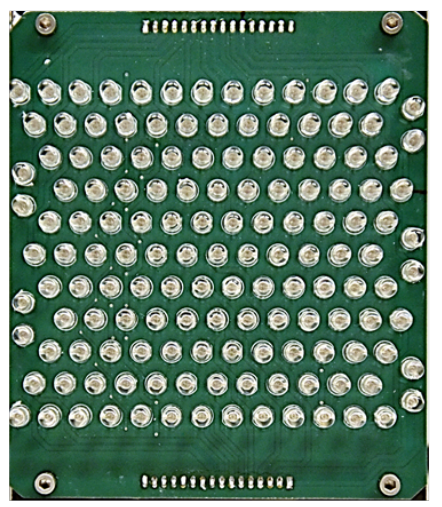

CC-8mm: 84 LEDs

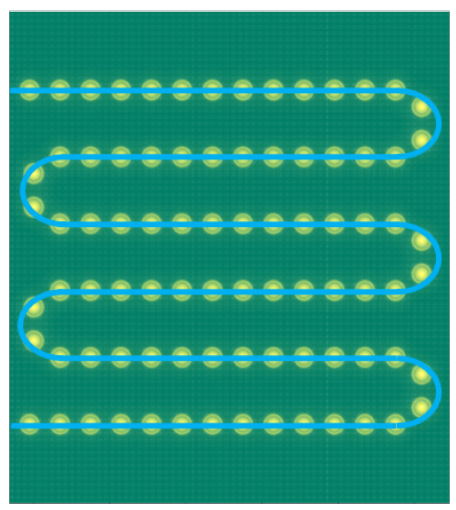

CC-6.5mm: 102 LEDs

b)
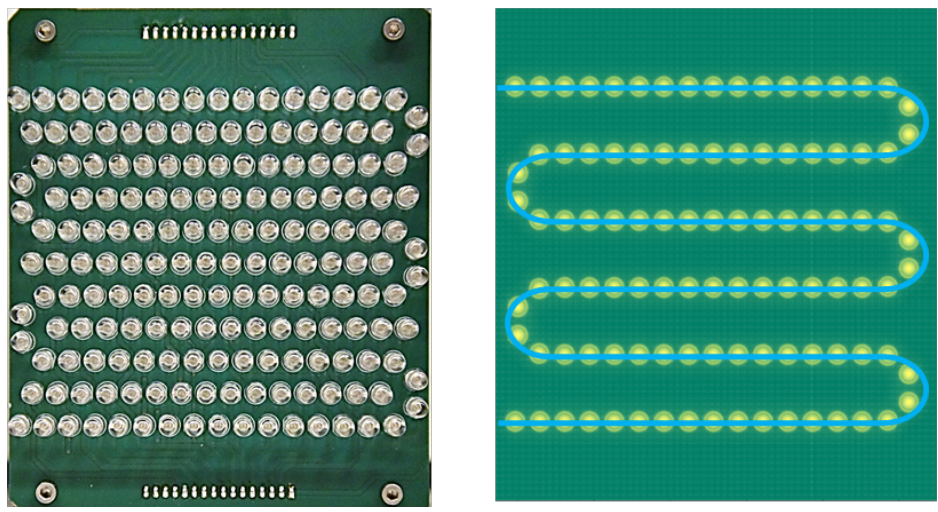

MC-8mm: 144 LEDs

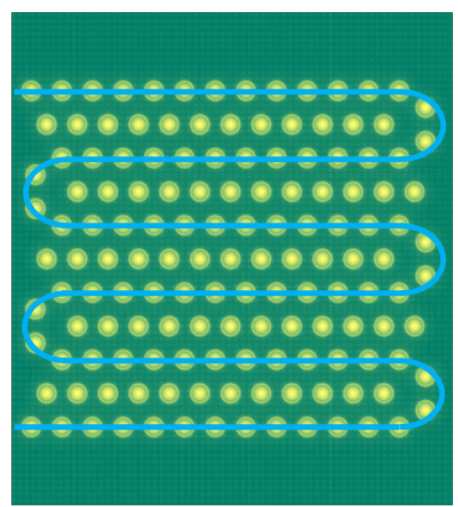

MC-6.5mm: 177 LEDs

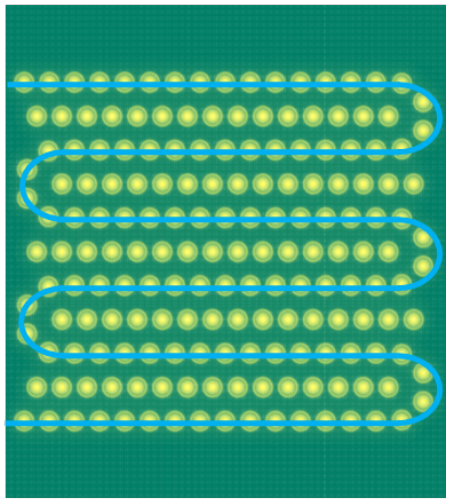

Figure 7. a) Photograph of the LED board with a LED-LED distance, $s$, of $8 \mathrm{~mm}$ which can be powered as channel configuration, CC-8mm, or matrix configuration, MC-8mm. b) Photograph of the LED board with a LED-LED distance, $s$, of $6.5 \mathrm{~mm}$ which can be powered as channel configuration, CC-6.5mm, or matrix configuration, MC-6.5mm. The relative position of the microchannel in the reactor is illustrated with a blue line (width not to scale).

\subsubsection{Optical and electrical properties}

LEDs employed in this study are characterized by a monochromatic emission in the green wavelength region as it can be observed in Fig. 8. By increasing the forward current from $2 \mathrm{~mA}$ to $25 \mathrm{~mA}$ the full width at half maximum (FWHM) of the emission increases from 30 to $32 \mathrm{~nm}$ and its maximum wavelength shifts from $526 \mathrm{~nm}$ to $520 \mathrm{~nm}$ (see Fig. S6). 


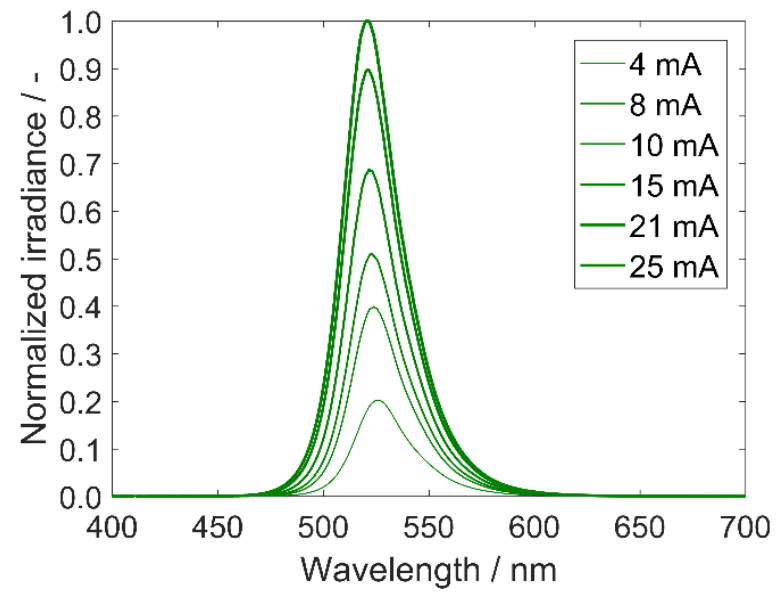

Figure 8. Variation of LED spectral distribution at different forward currents, $I_{F}$.

However, we observed that LEDs acquired in a single batch from the same supplier exhibited different light intensities when operated at the same voltage (see inset in Fig. 9a). This could be due to imperfections in the materials and variability in manufacturing ${ }^{23}$. Based on their light output, the analyzed LEDs were divided in three groups and denoted as LED1, LED2 and LED3 (increasing intensity from LED1 to LED3). Their forward current/forward voltage characteristic was measured and an example from each group is illustrated in Fig. 9a. A significant higher current is observed through the LED3 emitters in comparison with LED1 emitters at the same applied voltage, leading to a higher light output for the former.

a)

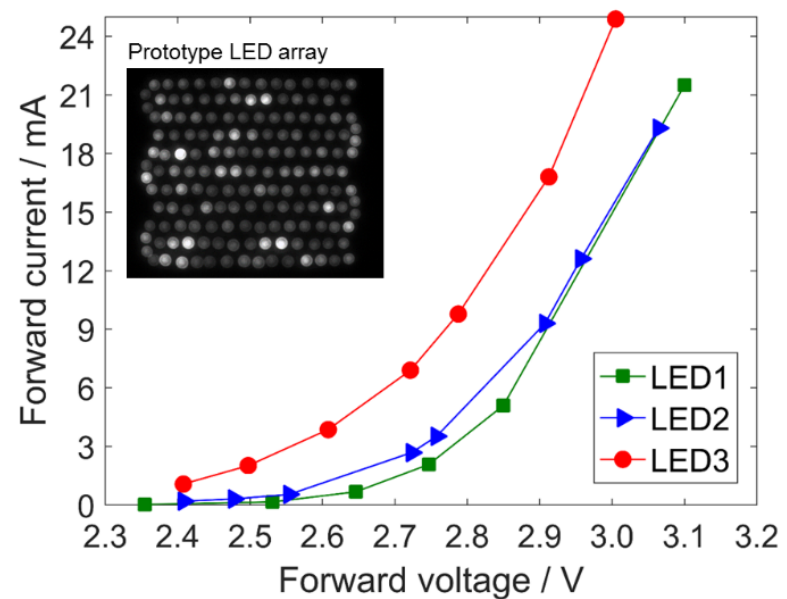

b)

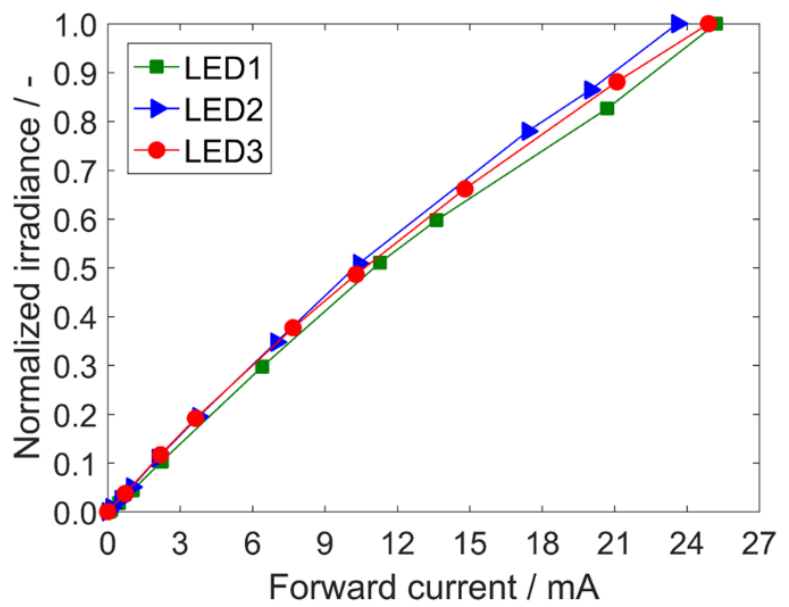

Figure 9. a) The dependence of the forward current, $I_{F}$, in function of the forward voltage, $V_{F}$, for LEDs representing the LED1, LED2, LED3 groups. In the inset, the first LED board prototype which contained LEDs with different intensities is pictured. b) Variation of normalized irradiance in function of the forward current, $I_{F}$, for LEDs representing the LED1, LED2, LED3 groups. 
Figure $9 \mathrm{~b}$ shows the variation of irradiance in function of the forward current, $I_{F}$, for the three groups of LEDs. A linear increase of the irradiance with the forward current is observed for all LEDs until $10 \mathrm{~mA}$. Therefore, to design a LED array with uniform irradiation requires to establish a similar current through all LEDs in the array. To achieve this, a driving board was designed which controls the current through series of LEDs using a current mirror. The description of the LED board design and operation are detailed in section 7 in the ESI.

\subsubsection{Thermal management}

The thermal management of LEDs is another important aspect to consider when designing LED light sources, as the LED junction temperature can affect the forward voltage (optical output) and the emission wavelength. ${ }^{23-25}$ Moreover, prolonged operation of LEDs at high temperatures leads to a reduction of their lifetime. ${ }^{23,26}$ Consequently, the LED board should be operated under isothermal conditions in order to maintain a constant optical output. For the LEDs used in this work, the operating temperature ranges from -45 to $85^{\circ} \mathrm{C}$ according to the manufacturer datasheet, while the majority of the optical properties in the datasheet are given for a junction temperature of $25^{\circ} \mathrm{C}^{13}$. However, it is difficult to determine the junction temperature and usually the surface temperature (i.e. on the polymeric encapsulation) is monitored. To compare the temperature at the surface of the LED and the temperature in the proximity of the junction, thermal images of an unmodified LED and a LED with the polymeric tip cut off were acquired (see Fig. 10a). A stepwise increase of the forward current from 11 to $31 \mathrm{~mA}$ results in a linear increase of the LED temperature. However, while the temperature at the surface of the unmodified LED increases by about $15^{\circ} \mathrm{C}$, the temperature increase in the proximity of the LED junction is more than twice this value (see Fig.10b). Therefore, moderate temperatures measured at the surface of the LEDs with encapsulation mean elevated temperatures near the LED junction, especially when operated at large forward currents. 
a)

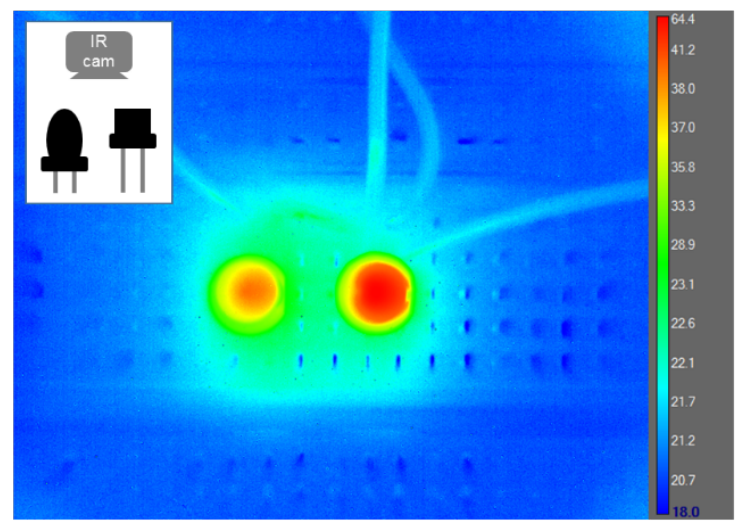

b)

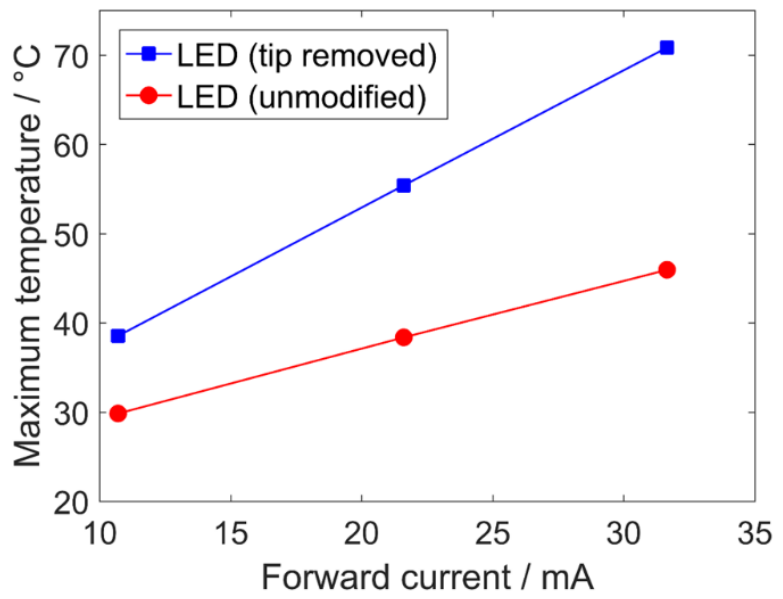

Figure 10. a) Temperature profile map acquired with an IR camera after reaching thermal equilibrium after 5 min of an unmodified LED (left) and a LED with the polymeric tip cut off, representing temperatures in the proximity of the junction (right). The forward current through the LEDs is $21 \mathrm{~mA} / \mathrm{LED}$. b) Variation of maximum temperature in function of forward current for the LED surface (unmodified) and the proximity of the LED junction (tip removed). The temperature values are extracted after reaching thermal equilibrium $(5 \mathrm{~min})$ and are averaged over three replicates (error bars are present but do not exceed the marker size).

a)
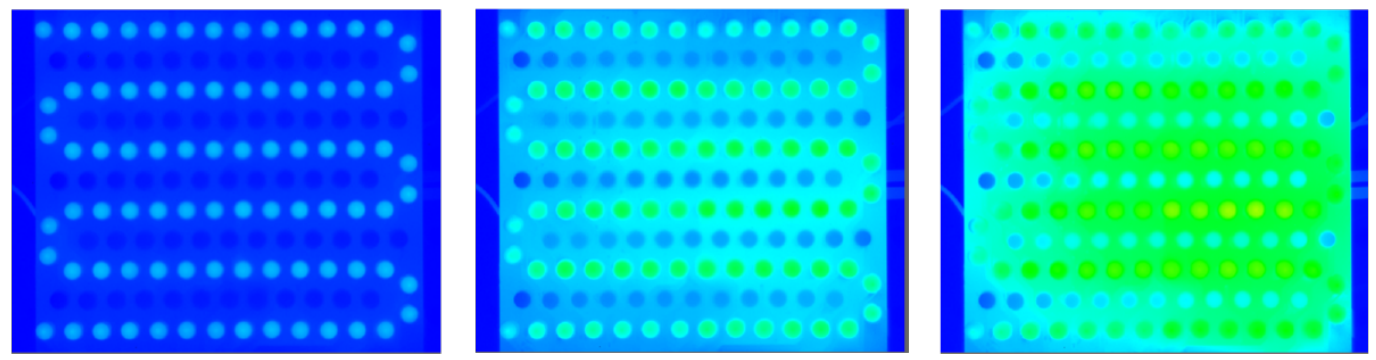

$1 \mathrm{~min}$

b)

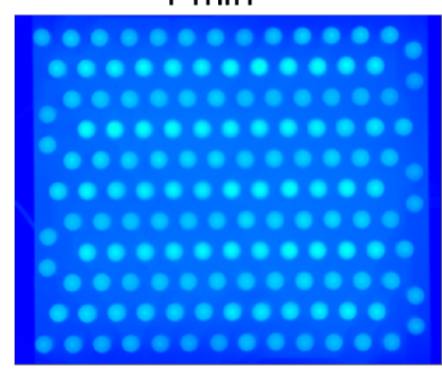

$1 \mathrm{~min}$

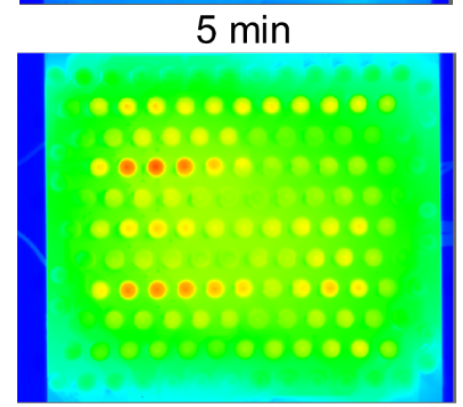

$5 \min$

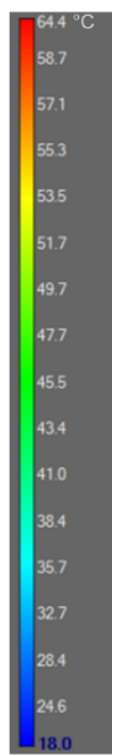

$6.3 \mathrm{~min}$

Figure 11. Temperature maps obtained with an IR camera over time for 2 different LED array configurations: a) CC-8mm and b) MC-8mm. The LED boards were operated at the forward current, $I_{\mathrm{F}}$, of $21 \mathrm{~mA} / \mathrm{LED}$ and were not equipped with a cooling system. 
Furthermore, if the LEDs are placed next to each other on a board their temperature will be higher in comparison with a single LED. To investigate the temperature of the LED boards in dependence of the number of illuminated LEDs, thermal images of the CC-8mm and MC-8mm configuration without active cooling were obtained and are shown in Fig. 11. In the CC-8mm configuration the temperature distribution of the LED array can be considered homogeneous over time with a maximum temperature of $47^{\circ} \mathrm{C}$ after $20 \mathrm{~min}$. Contrary to this, operating the $\mathrm{MC}-8 \mathrm{~mm}$ configuration with 60 more LEDs compared to CC-8mm results in inhomogeneities in the temperature distribution and hot spots reaching $60^{\circ} \mathrm{C}$ after only $5 \mathrm{~min}$. After $6.3 \mathrm{~min}$, the number of LEDs reaching $60^{\circ} \mathrm{C}$ has significantly increased especially in the center of the board, and the operation was stopped. This clearly illustrates that increasing the number of LEDs requires a cooling system to efficiently remove the heat.

As addressed earlier, this type of LEDs has an epoxy encapsulation which serves as a lens for the emitted light, but this encapsulation also represents a major resistance to heat transfer. Figure 12a compares the temperature maps during operation of a unmodified LED and a LED with the polymeric tip cut off along the metal leads. As it can be seen, the heat produced around the junction is mainly conducted through one of the leads, which is the cathode as shown in the schematic Fig. 12b. Therefore, convective cooling using an air flow over the LED surfaces would not efficiently cool the LED array. For an effective cooling, a thermal paste, which is electrically non-conductive, was used to connect the metal leads of each LED with a heat sink, which was cooled by convection using a fan.

a)

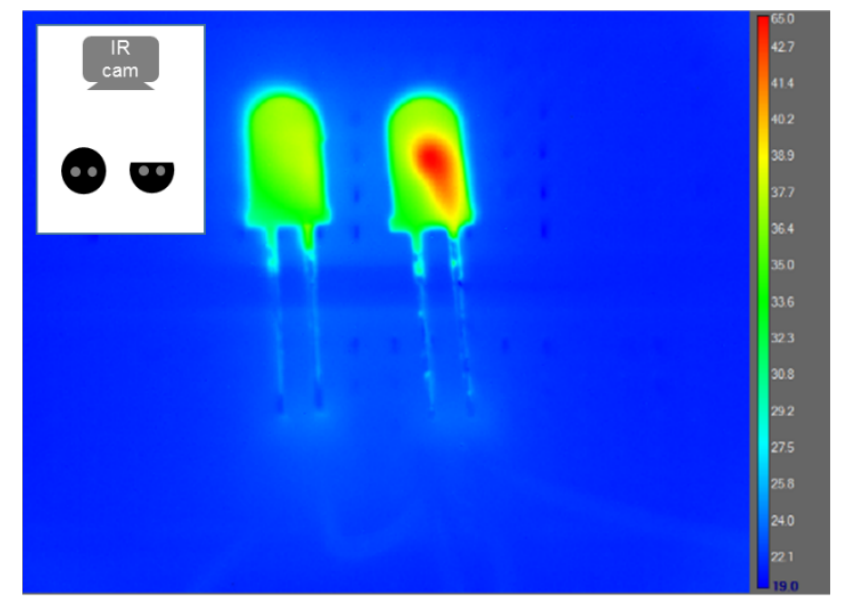

b)

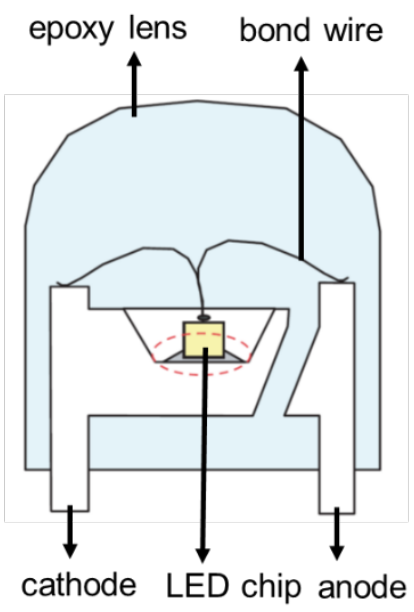

Figure 12. a) Temperature map acquired with an IR camera after reaching thermal equilibrium ( $5 \mathrm{~min}$ ) of an unmodified LED (left) and a LED with the polymeric tip cut off along the metal leads, representing temperatures in the proximity of the junction (right). The forward current through the LEDs, $I_{F}$, is $21 \mathrm{~mA} / L E D$. b) Schematic representation of the LEDs components adapted from the manufacturer datasheet ${ }^{13}$. 


\subsection{Characterization of LED light sources}

\subsubsection{Irradiance uniformity}

Another important metric is the irradiance uniformity on the reactor channel surface, as the chemical reaction only occurs inside the microchannel. The irradiance uniformity, $U$, is calculated as the ratio of the averaged irradiance and the maximum irradiance on the microchannel surface:

$$
U=\frac{\frac{1}{N} \sum_{N} E_{i}(x, y)}{\max \left[E_{i}(x, y)\right]}
$$

where $U$ is the irradiance uniformity [\%], $E_{\mathrm{i}}(x, y)$ is the simulated irradiance value on a point located on the microchannel surface $\left[\mathrm{W} \mathrm{cm}{ }^{-2}\right]$ and $N$ is the number of irradiance values simulated on the channel surface. The irradiance was calculated using the model (NFGM).

The obtained uniformity values are plotted in Fig.13 for the 4 LED array configurations as a function of the distance, $D$, between the LEDs and the reactor surface. Initially, the uniformity increases with increasing distance until it reaches a maximum. The distance of this maximum depends on the LED array configuration. In the case of CC-6.5mm the maximum uniformity is $92 \%$ at $D=1 \mathrm{~cm}$, while a uniformity of $91 \%$ is achieved at $D=1.5 \mathrm{~cm}$ for CC-8mm. This is explained by the smaller spatial separation between individual LEDs, for which a shorter distance is necessary for the emitted light to interact. Interestingly, for both MC configurations the maximum uniformity is obtained at the same distances as for the corresponding CC arrays, but with a decreased uniformity: $88 \%$ for MC-6.5mm and $86 \%$ for MC-8mm. This lower uniformity can be explained by considering the distribution of the light on the reactor plate.

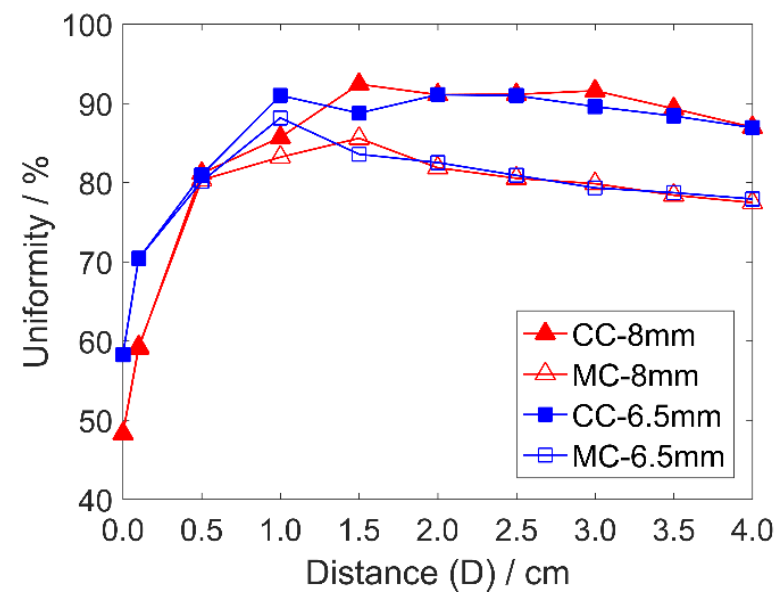

Figure 13. Variation of the irradiance uniformity, $U$, on the reactor channel in function of the distance, $D$, between the LEDs and the reactor surface. The irradiance uniformity was calculated based on the simulated irradiance values using model (NFGM).

Figure 14 shows the irradiance determined for the $\mathrm{CC}-8 \mathrm{~mm}$ and $\mathrm{MC}-8 \mathrm{~mm}$ arrays on a planar surface placed at $D=2$ and $4 \mathrm{~cm}$. The microchannel is represented with a black line, and irradiance profiles are extracted in the top 3 straight sections (denoted as S1, S2, S3 in Fig. 14). 
a)

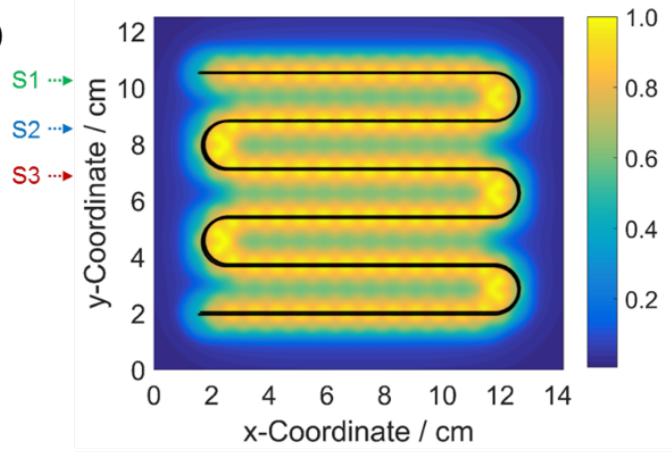

b)

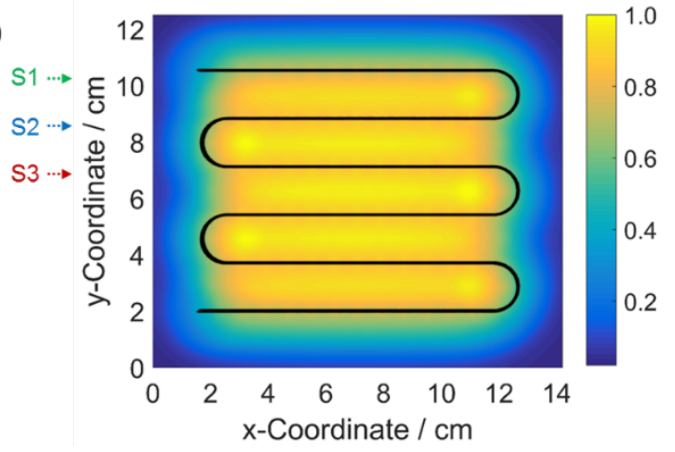

c)

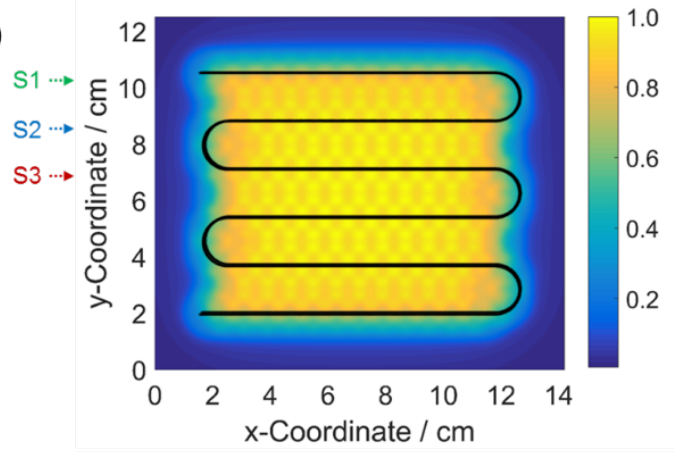

d)

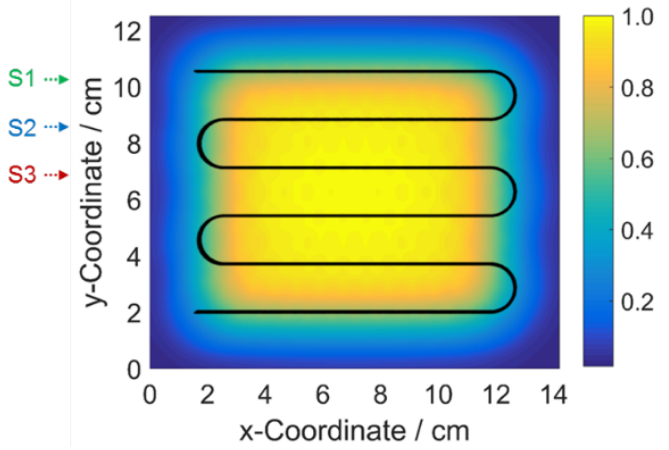

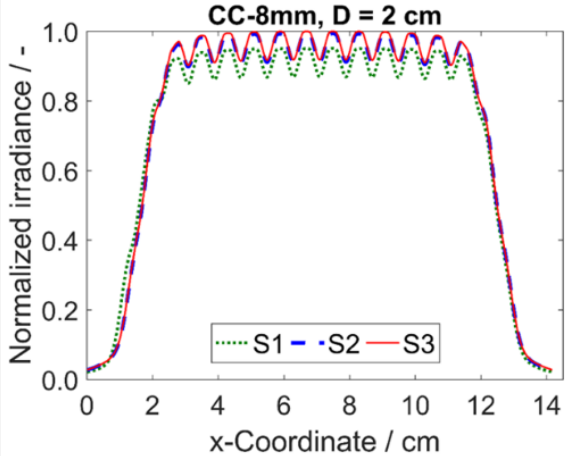

CC-8mm, $D=4 \mathrm{~cm}$
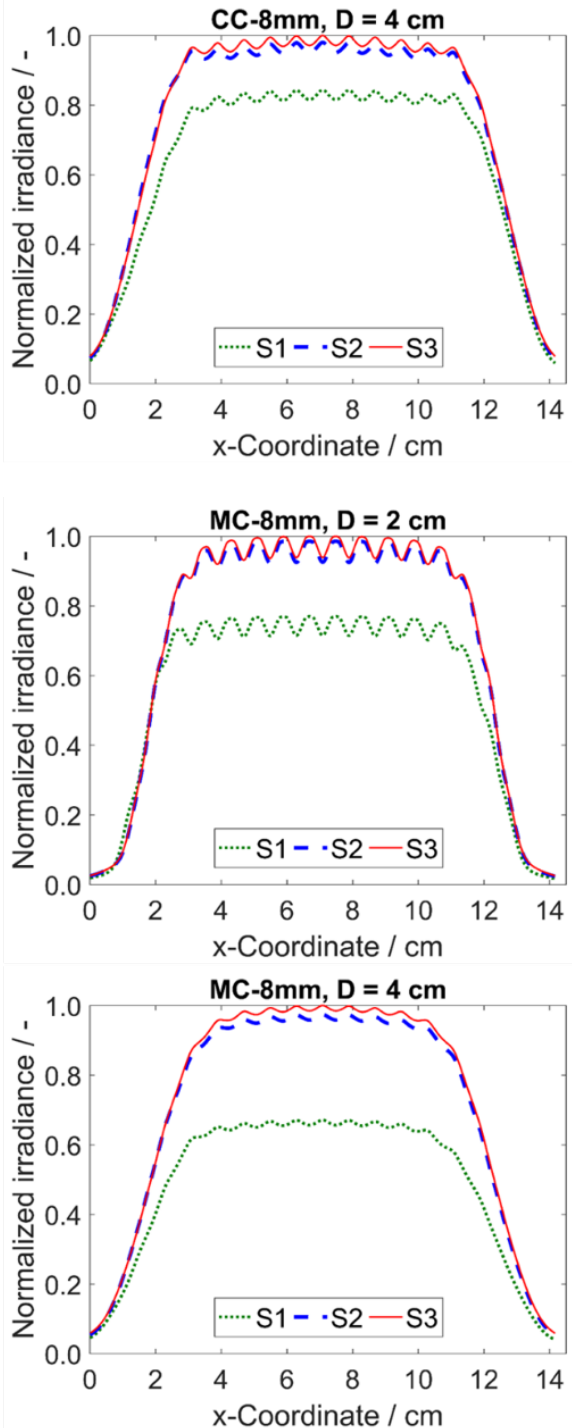

Figure 14. Comparison of the irradiance on a parallel plane placed at a) $D=2 \mathrm{~cm}$ for CC-8mm, b) $D=4 \mathrm{~cm}$ for CC-8mm, c) $D=2 \mathrm{~cm}$ for MC-8mm and d) $D=4 \mathrm{~cm}$ for MC-8mm. The location of the reactor channel is represented with a back line. The irradiance values were simulated using model (NFGM). For each case, the irradiance profiles on three reactor channel sections (S1-S3) are extracted and plotted in function of the $x$-Coordinate. 
As shown in Fig. 14a, the CC-8mm array results in similar irradiance values in these 3 sections, leading to an uniformity of $91 \%$ at $D=2 \mathrm{~cm}$. At the same distance, a uniformity of $86 \%$ is determined for the MC-8mm array, as the central sections (S2 and S3) experience a higher irradiance compared to the outer one (S1). At this distance, the LEDs located between the microchannel sections contribute to the irradiance on the microchannel. Therefore, the outer section experiences a lower irradiance because it receives the light only from one additional row of LEDs. When increasing the distance between the LEDs and the reactor to $4 \mathrm{~cm}$ (see Fig. 14b,d), the irradiance becomes more homogeneous for the center of each section, but the uniformity on the entire reactor channel decreases to $87 \%$ for CC-8mm and $78 \%$ for $\mathrm{MC}-8 \mathrm{~mm}$. The unequal illumination of section S1 compared with sections S2 and S3 for MC-8mm is accentuated at this distance, and is also observed for CC-8mm.

\subsubsection{Photon flux}

The photon flux, $I_{0}$, received in the microchannel under irradiation with the 4 LED arrays was determined with actinometry, and is plotted in Fig. 15 subject to the distance $D$. As expected, the amount of photons reaching the microchannel decreases with distance. However, in the considered range between $D=0 \mathrm{~cm}$ and $D=4 \mathrm{~cm}$ the decrease in the photon flux for CC configurations is around $66 \%$, but only around $47 \%$ for the MC configurations. When increasing $D$ the gradual contribution of the additional LEDs placed in the MC arrays maintains a high amount of photons in the microchannel, which is an opposite trend as found for the irradiance uniformity. Moreover, the photon flux scales with the number of LEDs in the array for $D>1 \mathrm{~cm}$, i.e. the highest photon flux is found for MC-6.5mm which contains 177 LEDs. This changes for distances $D<1 \mathrm{~cm}$ between the LEDs and the reactor surface, as a higher photon flux is observed for the CC-6.5mm (102 LEDs) compared to MC-8mm which contains 144 LEDs. At these short distances, the light emitted from the additional LEDs located between the microchannel sections in the MC configuration is no longer directly irradiating the reactor channel. However, the larger photon flux found for the $\mathrm{MC}$ configurations at $D=0 \mathrm{~cm}$ compared to the $\mathrm{CC}$ configurations suggests that rays emitted from these additional LEDs reach the microchannel via scattering and internal reflection in the reactor glass plate. 


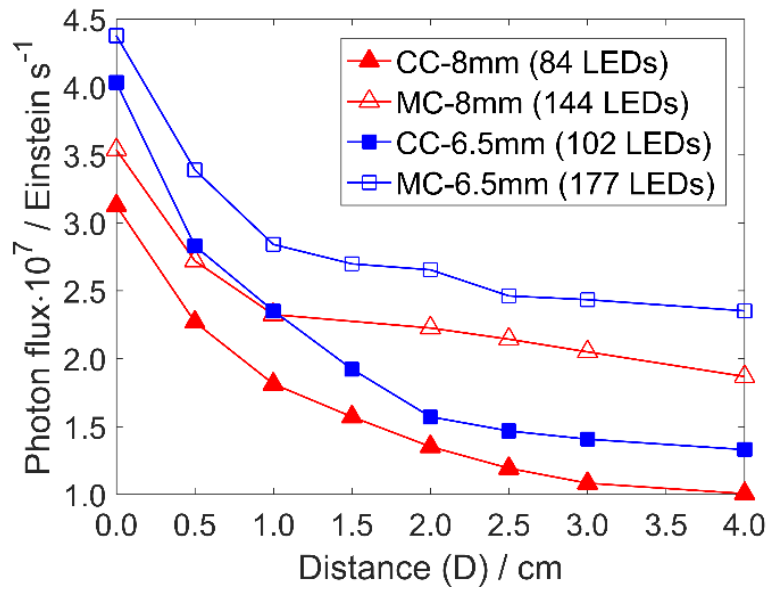

Figure 15. Variation of the photon flux, $I_{0}$, received in the microchannel with the distance, $D$, for CC- $8 \mathrm{~mm}, \mathrm{MC}-8 \mathrm{~mm}, \mathrm{CC}-6.5 \mathrm{~mm}$ and MC- $-6.5 \mathrm{~mm}$ arrays. $D$ is the distance between the LEDs and microstructured photoreactor. The actinometric measurements were performed at a forward current, $I_{F}$, of $8 \mathrm{~mA} / L E D$.

\subsubsection{Efficiency of the LED light sources}

The efficiency of the light sources is especially important for processes implementing continuous microreactors, as these processes are usually scaled-out by parallelization of multiple photoreactors. In order to assess the reactor performance with respect to the utilized LED array, a recent benchmark called the photochemical space-time yield (PSTY) was used. ${ }^{5}$ PSTY [mol W $\mathrm{W}^{-1} \mathrm{~s}^{-1}$ ] is the ratio of space-time yield, STY, to the lamp power normalized to the reactor volume, LP:

$$
\text { PSTY }=\frac{\text { STY }}{\text { LP }}
$$

In this work, the STY [mol L-1 $\mathrm{s}^{-1}$ ] was assumed equal to the averaged reaction rate in the reactor, as the overall conversion in the reactor is low $(<10 \%)$. The derivation of the averaged reaction rate expression is provided in section 8 of the ESI.

$$
\mathrm{STY}=\bar{R}=\phi_{\mathrm{avg}} \frac{I_{0}}{V}
$$

where $\phi_{\text {avg }}$ is the averaged quantum yield which is equal to $0.02 \mathrm{~mol} \mathrm{Einstein}^{-1}$ at $I_{\mathrm{F}}=8 \mathrm{~mA} / \mathrm{LED}$ as shown in Fig. S1.3, and $V$ is the reactor volume [L]. The standardized lamp power, LP [W L ${ }^{-1}$ ], was calculated as: 


$$
\mathrm{LP}=\frac{P}{V}
$$

where $P$ is the power consumed by the light source which includes the driving and LED boards [W].

Figure 16a shows that the CC design is more efficient than the MC configurations at short LEDreactor distances. On the other hand, the MC arrays become more efficient when $D$ is larger than $2 \mathrm{~cm}$. This is due to the contribution of the LEDs located between microchannel sections to the photon flux with increasing distance. Furthermore, it is interesting to note that at most distances CC-6.5mm proved to be more efficient compared to CC-8mm, and MC- $6.5 \mathrm{~mm}$ was more efficient than $\mathrm{CC}-8 \mathrm{~mm}$. As the $\mathrm{CC}$ and $\mathrm{MC}$ designs provide a similar photon flux per LED irrespective of $D$ (see Fig. 16b), this improved efficiency for the smaller inter-LED spacing originates from the increased ratio of the power consumed by LEDs and the power consumed by the driving board. The latter remains fairly constant irrespective of the layout of the LED array, and therefore a larger fraction of the electricity is converted into photons with an increasing number of LEDs in the array.

a)

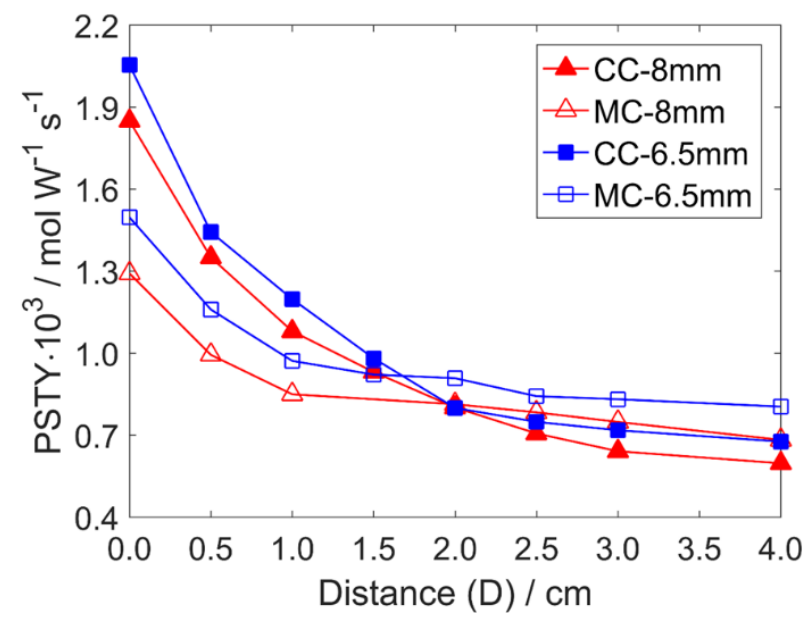

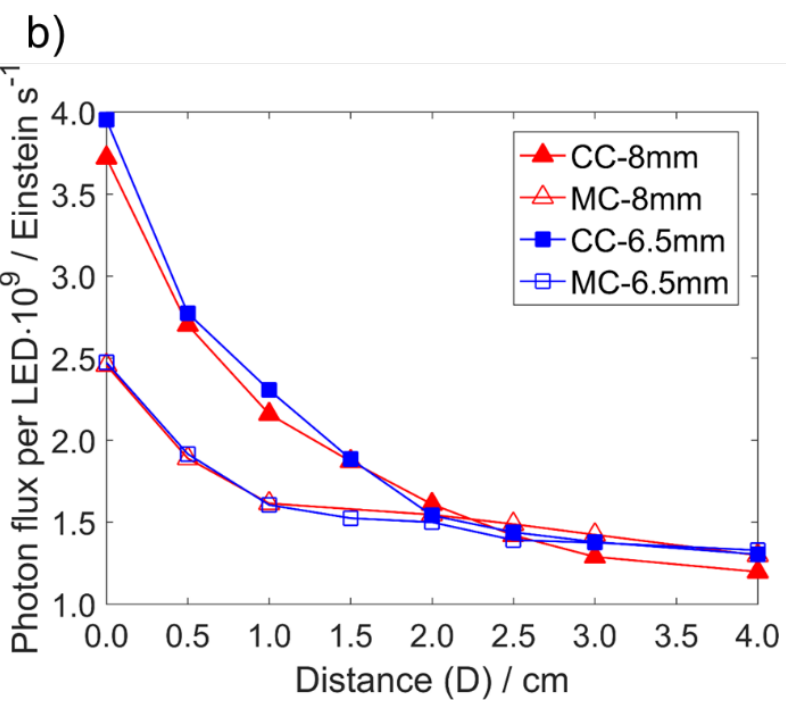

Figure. 16 a) Variation of PSTY in function of distance, D. b) Variation of the photon flux normalized by the number of LEDs contained in the light sources in function of distance, $D$. The actinometric measurements were performed at the forward current, $l_{\mathrm{F}}$, of $8 \mathrm{~mA} / L E D$.

Table 2 details the energy losses of the CC-8mm and $\mathrm{MC}-8 \mathrm{~mm}$ light sources when they were placed at $D=1 \mathrm{~cm}$ starting from the conversion of electricity into photons and their transport into the microchannel. The individual contributions to the energy consumption were investigated using (i) NFGM to measure the radiant flux emitted by the LED boards (see section 5 of the ESI); (ii) the irradiance model to determine the absolute irradiance of the LED arrays on the reactor surface (see section 5 of the ESI); and (iii) actinometry to determine the radiant flux received in the microchannel from measurements of the photon flux (see section 3 of the ESI). As shown in Tab. 2, up to $44 \%$ of the energy is consumed by the driving board to control the CC-8mm array. As previously discussed, the percentage is lower for MC- $8 \mathrm{~mm}$, due to the larger number of LEDs 
present on the board. Furthermore, only $30 \%$ (CC-8mm), respectively $27 \%$ (MC-8mm) of the electricity used to power the LEDs was converted to photons, a finding in accordance with reported LED efficiencies. ${ }^{4,27}$ The radiant fluxes arriving on top of the glass plate surface of the reactor $(10.6 \mathrm{~cm} \times 11.2 \mathrm{~cm})$ account for $17 \%$ of the consumed power. This highlights the advantage of using LEDs with narrow viewing angle, as only $7 \%$ of the incident photon flux was lost. However, only $2.5 \%$ (CC-8mm), respectively $1.6 \%(\mathrm{MC}-8 \mathrm{~mm})$ of the consumed power reaches the surface of the microchannel due to its small surface area $(0.1 \mathrm{~cm} \times 69.95 \mathrm{~cm})$. As it can be observed, in order to achieve a similar photon flux through the surface of the microchannel ( $85 \mathrm{~mW}$ ), MC-8mm consumes $62 \%$ more power than CC-8mm. Finally, only around $1 \%$ of the consumed power arrives into the reactor channel as photons. The main factors leading to this low utilization of the electric energy and which can be improved through photoreactor design are the high energy consumption of the driving board and the low surface area of the reactor exposed to the irradiation. These findings are essential for improving the design of photoreactors illuminated by LED light sources.

Table 2. Power utilization of the CC-8mm and MC-8mm light sources placed at a distance of $D=1 \mathrm{~cm}$. The light sources were operated at a forward current, $I_{\mathrm{F}}$, of $8 \mathrm{~mA} / L E D$. The power consumed by the LEDs is calculated considering an average voltage of $2.8 \mathrm{~V}$ at $8 \mathrm{~mA} / \mathrm{LED}$.

\begin{tabular}{|l|l|l|}
\hline Steps & CC-8mm & MC-8mm \\
\hline Power consumed by LED and driving boards, $P$ (measured) & $100 \%$ & $100 \%$ \\
& $(3375 \mathrm{~mW})$ & $(5475 \mathrm{~mW})$ \\
\hline Power consumed by LEDs (calculated) & $\begin{array}{l}55.61 \% \\
(1877 \mathrm{~mW})\end{array}$ & $\begin{array}{l}62.76 \% \\
(3436 \mathrm{~mW})\end{array}$ \\
\hline Radiant flux emitted by LEDs (measured) & $16.53 \%$ & $16.73 \%$ \\
& $(558 \mathrm{~mW})$ & $(916 \mathrm{~mW})$ \\
\hline Radiant flux through plate reactor surface (simulated) & $15.38 \%$ & $15.67 \%$ \\
& $(519 \mathrm{~mW})$ & $(858 \mathrm{~mW})$ \\
\hline Radiant flux through reactor channel (simulated) & $2.49 \%$ & $1.55 \%$ \\
& $(84 \mathrm{~mW})$ & $(85 \mathrm{~mW})$ \\
\hline Radiant flux received in the reactor channel, $I_{0}$ (measured) & $1.21 \%$ & $0.97 \%$ \\
& $(41 \mathrm{~mW})$ & $(53 \mathrm{~mW})$ \\
\hline
\end{tabular}

\section{Conclusions}

With the increasing use of LED light sources for irradiating microstructured photoreactors, guidelines for a rational design and a toolbox for their quantitative characterization are required for implementing controlled and efficient photochemical processes in flow. This study exemplifies how the optical and electrical properties of the LEDs can be translated into the LED array design and the choice of the driving mode. Moreover, the near-field goniophotometer measurements (NFGM) are used for the first time to extend the applicability of the irradiance model valid for the far-field. The improved model requires the angular irradiance distributions extracted from near-field measurements using a ray tracing software. With this adaptation, the distribution of the 
light on the reactor was accurately simulated and the absolute irradiance quantified for a range of distances between the LED array and the reactor. This method is advantageous as near-field measurements for a broad variety of LEDs are available free of charge on manufacturer's websites. In this work, four LED arrays based on narrow viewing angle LEDs of $30^{\circ}$ were designed and characterized using NFGM, irradiance modeling and actinometry. It was shown that the irradiance uniformity on the microchannel increased with the distance LED-reactor until reaching a maximum. The maximum uniformity of around $90 \%$ was achieved at $1 \mathrm{~cm}$ and $1.5 \mathrm{~cm}$ when the distance LED-LED was $6.5 \mathrm{~mm}$ and $8 \mathrm{~mm}$ respectively. At these small LED-reactor distances, adding additional LEDs between the microchannel sections led to an increase of the photon flux received by the microchannel while the uniformity and PSTY were reduced. At LED-reactor distances larger than $2 \mathrm{~cm}$, the emission of the additional LEDs contributes to the photon flux received by the microreactor, therefore the PSTY increased, however the uniformity continued to decline. Moreover, it was found that only about $1 \%$ of the power consumed by the light source (LED and driving board) was received in the microchannel as photons, irrespective of the LED array design. The characterization methodology presented in this work enabled the identification and quantification of the main parameters important for improving the energy use: (i) the energy efficiency of the driving board and (ii) the surface area of the reactor exposed to the LED radiation. Therefore, the designs of future microstructured photoreactors should continue focusing on improving the use of the incident photons. As shown in this study, novel photo-microreactors need to consider the interplay between the type of LEDs, their arrangement on a PCB, the geometry of the microreactor and the distance between the light source and the microreactor.

\section{List of Symbols}

\section{Roman letters}

$a_{i}, b_{i}, c_{i} \quad$ Equation coefficients to fit the angular irradiance distribution

$\Delta d \quad$ Distance between the LED chip and the LED tip (cm)

$D \quad$ Distance between the tip of the LED and the illuminated surface $(\mathrm{cm})$

$E(\theta) \quad$ Normalized angular irradiance distribution (-)

I Radiant intensity ( $\left.\mathrm{W} \mathrm{sr}^{-1}\right)$

$I(\theta) \quad$ Normalized angular intensity distribution (-)

$I(\theta)_{\mathrm{A}} \quad$ Absolute angular intensity distribution $\left(\mathrm{W} \mathrm{sr}^{-1}\right)$

$I_{F} \quad$ Forward current $(\mathrm{mA})$

$I_{\text {Max }} \quad$ Maximum radiant intensity for the polar angle $\theta=0$

$I_{0} \quad$ Photon flux received in the microchannel (Einstein $\mathrm{s}^{-1}$ )

$m \quad$ Parameter to account for the LED encapsulation 


$\begin{array}{ll}M & \text { Number of LEDs considered in the irradiance simulation } \\ N & \text { Number of simulated irradiance values } \\ P & \text { Total power consumed by the driving and LED boards }(\mathrm{W}) \\ r & \text { Arbitrary point on irradiated plane } \\ R & \text { Radial coordinate } \\ S & \begin{array}{l}\text { Ratio characterizing the broadening of the angular irradiance distribution } \\ \text { in the near-field }\end{array} \\ U & \text { Distance between two adjacent LEDs in the LED array (mm) } \\ V & \text { Irradiance uniformity (\%) } \\ V_{F} & \text { Reactor volume (L) } \\ x, y, z & \text { Forward voltage (V) } \\ x_{0}, y_{0} & \text { Cartesian coordinates }\end{array}$

\section{Greek letters}

$\begin{array}{ll}\theta & \text { Polar angle }\left(\mathrm{rad},{ }^{\circ}\right) \\ \theta_{1 / 2} & \text { Polar angle at which the maximum intensity decreases to } 50 \%\left(\mathrm{rad},{ }^{\circ}\right) \\ \phi & \text { Azimuthal angle }\left(\mathrm{rad},{ }^{\circ}\right) \\ \phi_{\text {avg }} & \text { Average quantum yield }\left(\text { mol Einstein }^{-1}\right) \\ \Phi_{0} & \text { Radiant flux (W) } \\ \Omega & \text { Solid angle (sr) }\end{array}$

\section{Abbreviations}

CC Channel configuration

CC-6.5mm LED array in channel configuration, $s=6.5 \mathrm{~mm}$ and 102 LEDs

CC-8mm LED array in channel configuration, $s=8 \mathrm{~mm}$ and 84 LEDs

CFL Compact fluorescent lamp

DAE CF 1,2-bis(2,4-dimethyl-5-phenyl-3-thienyl)perfluorocyclopentene closed form

DAE OF 1,2-bis(2,4-dimethyl-5-phenyl-3-thienyl)perfluorocyclopentene open form

DOM Discrete ordinate method 


$\begin{array}{ll}\text { DS } & \text { Manufacturer datasheet } \\ \text { FWHM } & \text { Full width at half maximum }(\mathrm{nm}) \\ \text { LED } & \text { Light-Emitting Diode } \\ \text { LP } & \text { Lamp power normalized to the reactor volume }\left(\mathrm{W} \mathrm{L}^{-1}\right) \\ \text { MC } & \text { Matrix configuration } \\ \text { MC-6.5mm } & \text { LED array in matrix configuration, } s=6.5 \mathrm{~mm} \text { and 177 LEDs } \\ \text { MC-8mm } & \text { LED array in matrix configuration, } s=8 \mathrm{~mm} \text { and 144 LEDs } \\ \text { NFGM } & \text { Near-field goniophotometer measurement } \\ \text { OLED } & \text { Organic Light-Emitting Diodes } \\ \text { PCB } & \text { Printed circuit board } \\ \text { PFA } & \text { Perfluoroalkoxy alkane } \\ \text { PSTY } & \text { Photochemical space-time yield }\left(\mathrm{mol} \mathrm{W}^{-1} \mathrm{~s}^{-1}\right) \\ \text { STY } & \text { Space-time yield }\left(\text { mol } \mathrm{L}^{-1} \mathrm{~s}^{-1}\right) \\ \text { UV } & \text { Ultraviolet }\end{array}$

\section{Conflicts of interest}

The authors have no conflicts to declare.

\section{Acknowledgement}

A.R. and S.K. would like to acknowledge the European Union for a Marie Curie ITN Grant (Photo4Future, grant number 641861). M.E.L. acknowledges FWO postdoctoral funding. D.K. acknowledges the State Secretariat for Education, Research and Innovation, Switzerland, within the Photo4Future Innovative Training Network, Grant No. 641861. We thank Sven Decock (Department of Electrical Engineering, KU Leuven) for helping with the design of the LED and driving board and Pieter Kramer (Laser 2000 Benelux) for helpful discussions.

\section{References}

1. J. P. Knowles, L. D. Elliott and K. I. Booker-Milburn, Beilstein J. Org. Chem., 2012, 8, 2025-2052.

2. S. G. Newman and K. F. Jensen, Green Chem., 2013, 15, 1456-1472.

3. D. Cambié, C. Bottecchia, N. J. W. Straathof, V. Hessel and T. Noël, Chem. Rev., 2016, 116, 10276-10341.

4. M. Sender and D. Ziegenbalg, Chem. Ing. Tech., 2017, 89, 1159-1173.

5. M. E. Leblebici, G. D. Stefanidis and T. Van Gerven, Chem. Eng. Process., 2015, 97, 106-111.

6. D. Ziegenbalg, B. Wriedt, G. Kreisel and D. Kralisch, Chem. Eng. Technol., 2016, 39, 123-134. 
7. L. H. Levine, J. T. Richards, J. L. Coutts, R. Soler, F. Maxik and R. M. Wheeler, J. Air Waste Manage. Assoc., 2011, 61, 932-940.

8. M. Martín-Sómer, C. Pablos, R. van Grieken and J. Marugán, Appl. Catal., B, 2017, 215, 1-7.

9. C. Casado, R. Timmers, A. Sergejevs, C. T. Clarke, D. W. E. Allsopp, C. R. Bowen, R. van Grieken and J. Marugán, Chem. Eng. J., 2017, 327, 1043-1055.

10. A. Kheyrandish, M. Mohseni and F. Taghipour, Water Res., 2017, 122, 570-579.

11. A. Kheyrandish, F. Taghipour and M. Mohseni, J. Photochem. Photobiol., A, 2018, 352, 113-121.

12. A. Sergejevs, C. T. Clarke, D. W. E. Allsopp, J. Marugan, A. Jaroenworaluck, W. Singhapong, P. Manpetch, R. Timmers, C. Casado and C. R. Bowen, Photochem. Photobiol. Sci., 2017, 16, 16901699.

13. The Mouser online datasheets www.mouser.com/ds/2/678/V02-2228EN DS HLMP-Cx1A 201305-220-909136.pdf, (accessed 19.07.2018).

14. A. Roibu, S. Fransen, M. E. Leblebici, G. Meir, T. Van Gerven and S. Kuhn, Sci. Rep., 2018, 8, 5421.

15. C.-C. Sun, T.-X. Lee, S.-H. Ma, Y.-L. Lee and S.-M. Huang, Opt. Lett., 2006, 31, 2193-2195.

16. I. Moreno and C.-C. Sun, Opt. Expres, 2008, 16, 1808-1819.

17. I. Moreno, C.-C. Sun and R. Ivanov, Appl. Opt., 2009, 48, 1190-1197.

18. S. Cool, J. G. Pieters, K. C. Mertens, S. Mora, F. Cointault, J. Dubois, T. van de Gucht and J. Vangeyte, Sensors, 2015, 15, 28627-28645.

19. I. Moreno, M. Avendaño-Alejo and R. I. Tzonchev, Appl. Opt., 2006, 45, 2265-2272.

20. J. J. B. Moerman and J. G. Holmes, Lighting Res. Technol., 1981, 13, 87-95.

21. V. Jacobs, S. Forment, P. Rombauts and P. Hanselaer, Lighting Res. Technol., 2015, 47, 470-482.

22. P. Manninen, J. Hovila, P. Kärhä and E. Ikonen, Meas. Sci. Technol., 2007, 18, 223-229.

23. M. Bürmen, F. Pernuš and B. Likar, Meas. Sci. Technol., 2008, 19, 122002-122017.

24. S. Chhajed, Y. Xi, T. Gessmann, J.-Q. Xi, J. M. Shah, J. K. Kim and E. F. Schubert, presented in part at the Proc of SPIE, Light-Emitting Diodes: Research, manufacturing, and applications IX, San Jose, California, United States, 2005.

25. E. Hong and N. Narendran, presented in part at the Proc of SPIE, Third international conference on solid state lighting, San Diego, California, United States, 2004.

26. N. Narendran, L. Deng, R. M. Pysar, Y. Gu and H. Yu, presented in part at the Proc. SPIE, Third international conference on solid state lighting, San Diego, California, United States, 2004.

27. D. A. Steigerwald, J. C. Bhat, D. Collins, R. M. Fletcher, M. O. Holcomb, M. J. Ludowise, P. S. Martin and S. L. Rudaz, IEEE J. Sel. Top. Quantum Electron, 2002, 8, 310-320. 